Network Working Group

Request for Comments: 2592

Category: Standards Track
D. Levi

Nortel Networks

J. Schoenwaelder

TU Braunschweig

May 1999

\title{
Definitions of Managed Objects for the Delegation of Management Scripts
}

\section{Status of this Memo}

This document specifies an Internet standards track protocol for the Internet community, and requests discussion and suggestions for improvements. Please refer to the current edition of the "Internet Official Protocol Standards" (STD 1) for the standardization state and status of this protocol. Distribution of this memo is unlimited.

Copyright Notice

Copyright (C) The Internet Society (1999). All Rights Reserved.

Abstract

This memo defines a portion of the Management Information Base (MIB) for use with network management protocols in the Internet community. In particular, it describes a set of managed objects that allow the delegation of management scripts to distributed managers.

Table of Contents

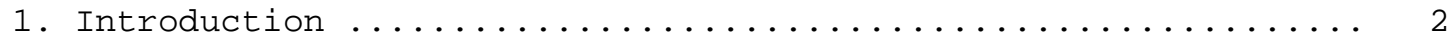

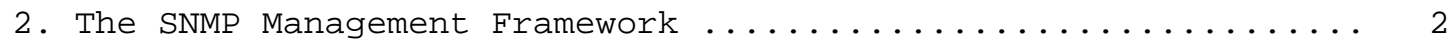

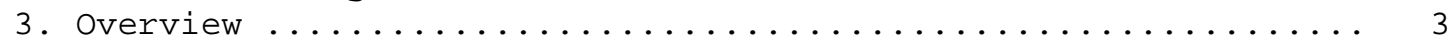

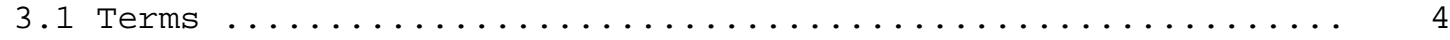

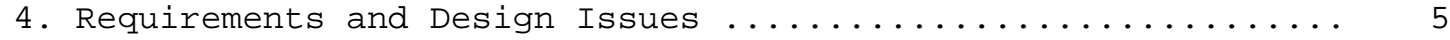

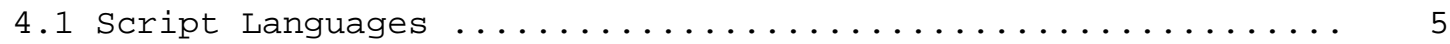

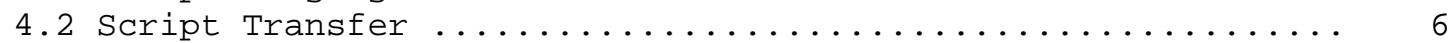

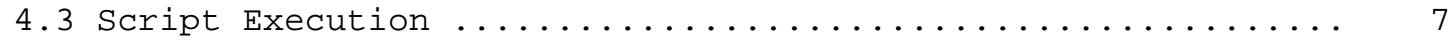

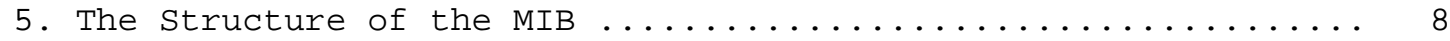

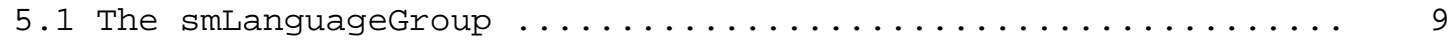

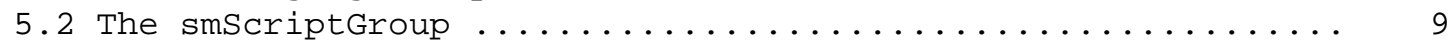

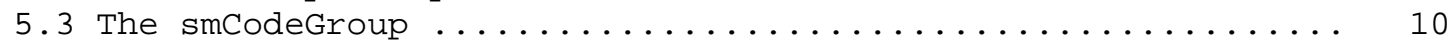

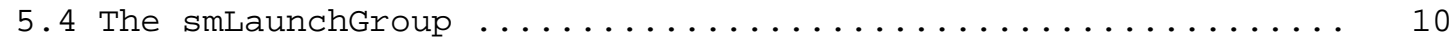

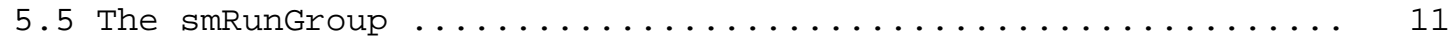

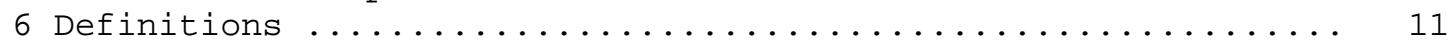

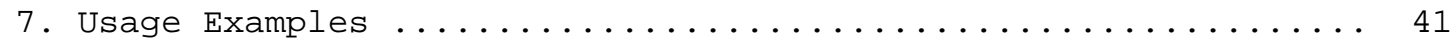

7.1 Pushing a script via SNMP $\ldots \ldots \ldots \ldots \ldots \ldots \ldots \ldots \ldots \ldots \ldots \ldots \ldots \ldots . \ldots \ldots \ldots$ 


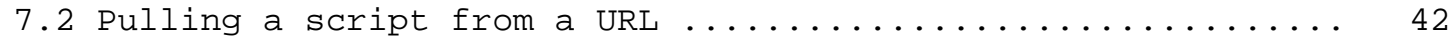

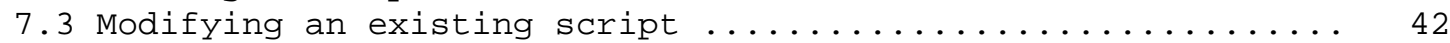

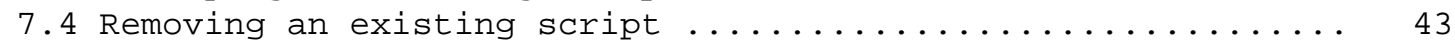

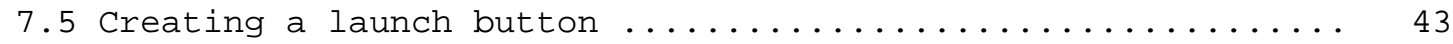

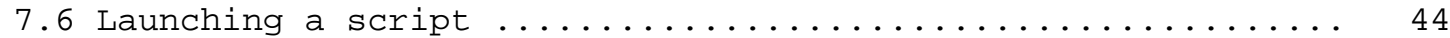

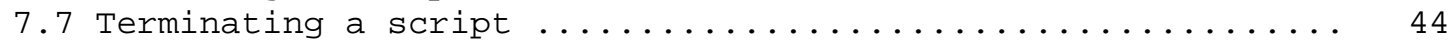

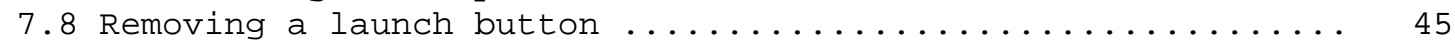

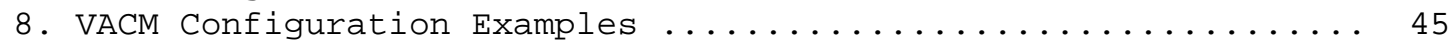

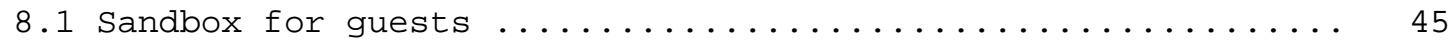

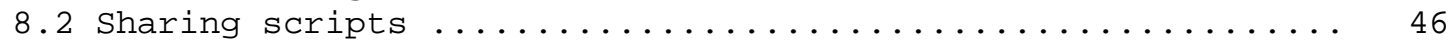

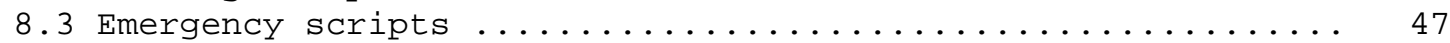

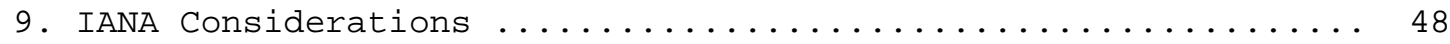

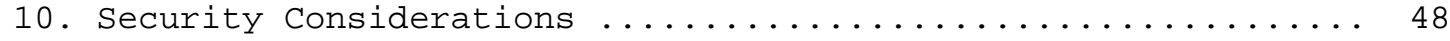

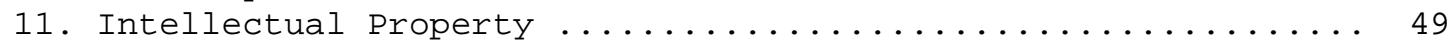

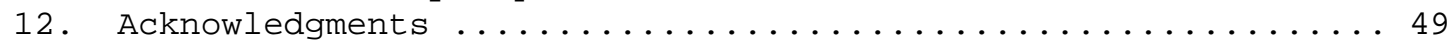

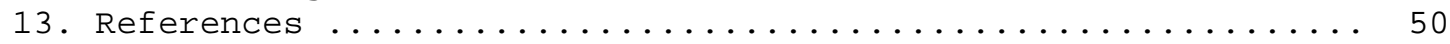

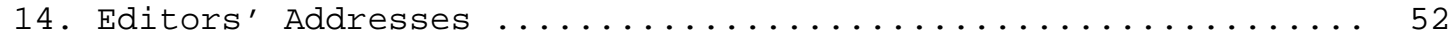

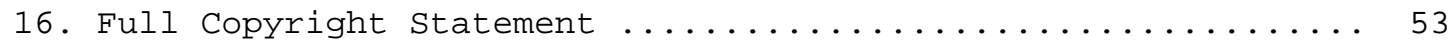

1. Introduction

This memo defines a portion of the Management Information Base (MIB) for use with network management protocols in the Internet community. In particular, it describes a set of managed objects that allow the delegation of management scripts to distributed managers.

The key words "MUST", "MUST NOT", "REQUIRED", "SHALL", "SHALL NOT", "SHOULD", "SHOULD NOT", "RECOMMENDED", "MAY", and "OPTIONAL" in this document are to be interpreted as described in RFC 2119 [21].

2. The SNMP Management Framework

The SNMP Management Framework presently consists of five major components:

- An overall architecture, described in RFC 2271 [1].

- Mechanisms for describing and naming objects and events for the purpose of management. The first version of this structure of Management Information (SMI) is called SMIv1 and described in STD 16, RFC 1155 [2], STD 16, RFC 1212 [3] and RFC 1215 [4]. The second version, called SMIV2, is described in STD 58, RFC 2578 [5], RFC 2579 [6] and RFC 2580 [7]. 
- Message protocols for transferring management information. The first version of the SNMP message protocol is called SNMPV1 and described in STD 15, RFC 1157 [8]. A second version of the SNMP message protocol, which is not an Internet standards track protocol, is called SNMPV2c and described in RFC 1901 [9] and RFC 1906 [10]. The third version of the message protocol is called SNMPv3 and described in RFC 1906 [10], RFC 2272 [11] and RFC 2274 [12].

- Protocol operations for accessing management information. The first set of protocol operations and associated PDU formats is described in STD 15, RFC 1157 [8]. A second set of protocol operations and associated PDU formats is described in RFC 1905 [13].

- A set of fundamental applications described in RFC 2273 [14] and the view-based access control mechanism described in RFC 2275 [15].

Managed objects are accessed via a virtual information store, termed the Management Information Base or MIB. Objects in the MIB are defined using the mechanisms defined in the SMI.

MIB conforming to the SMIv1 can be produced through the appropriate translations. The resulting translated MIB must be semantically equivalent, except where objects or events are omitted because no translation is possible (use of Counter64). Some machine readable information in SMIV2 will be converted into textual descriptions in SMIv1 during the translation process. However, this loss of machine readable information is not considered to change the semantics of the MIB.

\section{Overview}

The Script MIB module defined in this memo can be used to delegate management functions to distributed managers. Management functions are defined as management scripts written in a management scripting language. This MIB makes no assumptions about the language itself and even allows distribution of compiled native code, if an implementation is able to execute native code under the control of this MIB.

The Script MIB defines a standard interface for the delegation of management functions based on the Internet management framework. In particular, it provides the following capabilities:

1. Capabilities to transfer management scripts to a distributed manager. 
2. Capabilities for initiating, suspending, resuming and terminating management scripts.

3. Capabilities to transfer arguments for management scripts.

4. Capabilities to monitor and control running management scripts.

5. Capabilities to transfer the results produced by running management scripts.

This memo does not address any additional topics like the generation of notifications or how to address remote agents from a Script MIB implementation.

\subsection{Terms}

This section defines the terms used throughout this memo.

- A 'distributed manager' is a processing entity which is capable of performing network management functions. For the scope of this memo, a distributed manager is assumed to implement the Script MIB.

o A 'higher-level manager', or just 'manager', is a processing entity or human who initiates and controls the operations performed by one or more distributed managers.

- A 'management script' is a set of instructions written in an executable language which implements a management function.

- A 'management scripting language' is a language used to write management scripts. Note, the term scripting language does not imply that the language must have the characteristics of scripting languages (e.g. string orientation, interpretation, weak typing). The MIB defined in this memo also allows to control management scripts written in arbitrary compiled system programming languages.

- A 'distributed manager' can be decomposed into an 'SNMP entity' which implements the Script MIB defined in this memo and the 'runtime system' that executes scripts. The Script MIB sees the runtime system as the managed resource which is controlled by the MIB.

The runtime system can act as an SNMP application, according to the SNMP architecture defined in RFC 2271 [1]. For example, a runtime system which sends SNMP requests to other SNMP entities will act as a command generator application. The SNMP 
applications in the runtime system may use the same SNMP engine which also serves the command responder application used to implement the Script MIB, but they are not required to do so.

- A 'launch button' is the conceptual button used to start the execution of a management script. It assignes control parameters to a management script. In particular, it defines the ownership of the scripts started from a launch button. The ownership can be used by the language runtime system to enforce security profiles on a running management script.

4. Requirements and Design Issues

This section discusses some general requirements that have influenced the design of the script MIB.

- The Script MIB must not make any assumptions about specific languages or runtime systems.

- The Script MIB must provide mechanisms that help to avoid new management problems (e.g. script version problems) .

- The Script MIB must provide SNMP interfaces to all functions required to delegate management scripts. However, other protocols might be used in addition if they provide a significant improvement in terms of convenience for implementation or performance.

o The Script MIB must be organized so that access can be controlled effectively by using view-based access control [15].

The following sections discuss some design issues in more detail.

\subsection{Script Languages}

The Script MIB defined in this memo makes no assumption about the script language. This MIB can therefore be used in combination with different languages (such as Tcl or Java) and/or different versions of the same language. No assumptions are made about the format in which management scripts are transferred.

The Script MIB provides access to information about the language versions supported by a Script MIB implementation so that a manager can learn about the capabilities provided by an implementation. Languages and language versions are identified as follows: 
1. The language is identified by an object identifier. Object identifier for well-known languages will be registered by the Internet Assigned Numbers Authority (IANA). Enterprise specific languages can also be registered in the enterprise specific OID subtree.

2. A particular version of a language is identified by a language version number. The combination of a language object identifier and a language version is in most cases sufficient to decide whether a script can be executed or not.

3. Different implementations of the same language version might have differences due to ambiguities in the language definition or additional language features provided by an implementor. An additional object identifier value is provided which identifies the organization which provides the implementation of a language. This might be used by scripts that require a particular implementation of a language.

4. Finally, there might be different versions of a language implementation. A version number for the language implementation is provided so that the manager can also distinguish between different implementations from the same organization of a particular language version.

The version numbers can either be used by a manager to select the language version required to execute a particular script or to select a script that fits the language versions supported by a particular Script MIB implementation.

An additional table lists language extensions that provide features not provided by the core language. Language extensions are usually required to turn a general purpose language into a management language. In many cases, language extensions will come in the form of libraries that provide capabilities like sending SNMP requests to remote SNMP agents or accessing the local MIB instrumentation. Every extension is associated with a language and carries its own version numbers.

\subsection{Script Transfer}

There are two different ways to transfer management scripts to a distributed manager. The first approach requires that the manager pushes the script to the distributed manager. This is therefore called the 'push model'. The second approach is the 'pull model' where the manager tells the distributed manager the location of the script and the distributed manager retrieves the script itself. 
The MIB defined in this memo supports both models. The 'push model' is realized by a table which allows a manager to write scripts by sending a sequence of SNMP set requests. The script can be split into several fragments in order to deal with SNMP message size limitations.

The 'pull model' is realized by the use of Uniform Resource Locators (URLs) [17] that point to the script source. The manager writes the URL which points to the script source to the distributed manager by sending an SNMP set request. The distributed manager is then responsible for retrieving the document using the protocol specified in the URL. This allows the use of protocols like FTP [18] or HTTP [19] to transfer large management scripts efficiently.

The Script MIB also allows management scripts that are hard-wired into the Script MIB implementation. Built-in scripts can either be implemented in a language runtime system, or they can be built natively into the Script MIB implementation. The implementation of the 'push model' or the 'pull model' is not required.

Scripts can be stored in non-volatile storage. This allows a distributed manager to restart scripts if it is restarted (off-line restart). A manager is not required to push scripts back into the distributed manager after a restart if the script is backed up in non-volatile storage.

Every script is identified by an administratively assigned name. This name may be used to derive the name which is used to access the script in non-volatile storage. This mapping is implementation specific. However, the mapping must ensure that the Script MIB implementation can handle scripts with the same administrative name owned by different managers. One way to achieve this is to use the script owner in addition to the script name in order to derive the internal name used to refer to a particular script in non-volatile storage.

\subsection{Script Execution}

The Script MIB permits execution of several instances of the same or different management scripts. Script arguments are passed as OCTET STRING values. Scripts return a single result value which is also an OCTET STRING value. The semantic interpretation of result values is left to the invoking manager or other management scripts. A script invoker must understand the format and semantics of both the arguments and the results of the scripts that it invokes. 
Scripts can also export complex results through a MIB interface. This allows a management application to access and use script results in the same manner as it processes any other MIB data. However, the Script MIB does not provide any special support for the implementation of MIBs through scripts.

Runtime errors terminate active scripts. An exit code and a human readable error message is left in the MIB. A notification containing the exit code, the error message and a timestamp is generated when a script terminates with an error exit code.

Script arguments and results do not have any size limitations other than the limits imposed by the SMI and the SNMP protocol. However, implementations of this MIB might have further restrictions. A script designer might therefore choose to return the results via other mechanisms if the script results can be very large. One possibility is to return a URL as a script result which points to the file containing the script output.

Executing scripts have a status object attached which allows script execution to be suspended, resumed, or aborted. The precise semantics of the suspend and resume operations are language and runtime system dependent. Some runtime systems may choose to not implement the suspend/resume operations.

A history of finished scripts is kept in the MIB. A script invoker can collect results at a later point in time (offline operation). Control objects can be used to control how entries in the history are aged out if the table fills up.

5. The structure of the MIB

This section presents the structure of the MIB. The objects are arranged into the following groups:

- language group (smLanguageGroup)

o script group (smScriptGroup)

- script code group (smCodeGroup)

o script launch group (smLaunchGroup)

o running script group (smRunGroup) 


\subsection{The smLanguageGroup}

The smLanguageGroup is used to provide information about the languages and the language extensions supported by a Script MIB implementation. This group includes two tables. The smLangTable lists all languages supported by a Script MIB implementation and the smExtsnTable lists the extensions that are available for a given language.

\subsection{The smScriptGroup}

The smScriptGroup consists of a single table, called the smScriptTable. The smScriptTable lists all scripts known to a Script MIB implementation. The smScriptTable contains objects that allow the following operations:

o download scripts from a URL (pull model)

- read scripts from local non-volatile storage

o store scripts in local non-volatile storage

o delete scripts from local non-volatile storage

o list permanent scripts (that can not be changed or removed)

o read and modify the script status (enabled, disabled, editing)

A status object called smScriptoperStatus allows a manager to obtain the current status of a script. It is also used to provide an error indication if an attempt to invoke one of the operations listed above fails. The status change of a script can be requested by modifying the associated smScriptAdminstatus object.

The source of a script is defined by the smScriptsource object. This object may contain a URL pointing to a remote location which provides access to the management script. The script source is read from the smcodeTable (described below) or from non-volatile storage if the smScriptSource object contains an empty URL. The smScriptstorageType object is used to distinguish between scripts read from non-volatile storage and scripts read from the smcodeTable.

Scripts are automatically loaded once the smScriptAdminstatus object is set to 'enabled'. Loading a script includes retrieving the script (probably from a remote location), compiling the script for languages that require a compilation step, and making the code available to the runtime system. The smScriptoperstatus object is used to indicate the status of the loading process. This object will start in the 
state 'retrieving', switch to the state 'compiling' and finally reach the state 'enabled'. Errors during the retrieval or compilation phase will result in an error state such as 'compilationfailed'.

\subsection{The smCodeGroup}

The smCodeGroup consists of a single table, called the smcodeTable, which provides the ability to transfer and modify scripts via SNMP set requests. In particular, the smcodeTable allows the following operations:

- download scripts via SNMP (push model)

- modify scripts via SNMP (editing)

The smcodeTable lists the code of a script. A script can be fragmented over multiple rows of the smcodeTable in order to handle SNMP message size limitations. Modifications of the smcodeTable are only possible if the associated smScriptoperstatus object has the value 'editing'. The Script MIB implementation reloads the modified script code once the smScriptoperstatus changes to 'enabled' again.

The implementation of the smCodeGroup is optional.

\subsection{The smLaunchGroup}

The smLaunchGroup contains a single table, the smLaunchTable. An entry in the smLaunchiable represents a launch button which can be used to start a script. The smLaunchTable allows the following operations:

o associate a script with an owner used during script execution

- provide arguments and parameters for script invocation

o invoke scripts with a single set operation

The smLaunchtable describes scripts and their parameters that are ready to be launched. An entry in the smLaunchTable attaches an argument to a script and control values which, for example, define the maximum number of times that a script invoked from a particular row in the smLaunchtable may be running concurrently.

An entry in the smLaunchTable also defines the owner which will be used to associate permissions with the script execution. 


\subsection{The smRunGroup}

The smRunGroup contains a single table, called the smRunTable, which lists all scripts that are currently running or have terminated recently. The smRunTable contains objects that allow the following operations:

o retrieve status information from running scripts

o control running scripts (suspend, resume, abort)

o retrieve results from recently terminated scripts

- control the remaining maximum lifetime of a running script

o control how long script results are accessible

Every row in the smRunTable contains the argument passed during script invocation, the result produced by the script and the script exit code. The smRunTable also provides information about the current run state as well as start and end time-stamps. There are three writable objects in the smRunTable. The smRunLifeTime object defines the maximum time a running script may run before it is terminated by the Script MIB implementation. The smRunExpireTime object defines the time that a completed script can stay in the smRunTable before it is aged out. The smRunControl object allows running scripts to be suspended, resumed, or aborted.

6. Definitions

DISMAN-SCRIPT-MIB DEFINITIONS : := BEGIN

IMPORTS

MODULE-IDENTITY, OBJECT-TYPE, NOTIFICATION-TYPE,

Integer32, Unsigned32, mib-2

FROM SNMPV2-SMI

RowStatus, TimeInterval, DateAndTime, StorageType, Displaystring FROM SNMPV2-TC

MODULE-COMPLIANCE, OBJECT-GROUP, NOTIFICATION-GROUP FROM SNMPV2-CONF

SnmpAdminstring FROM SNMP-FRAMEWORK-MIB;

scriptMIB MODULE-IDENTITY

LAST-UPDATED "9902221800Z" 


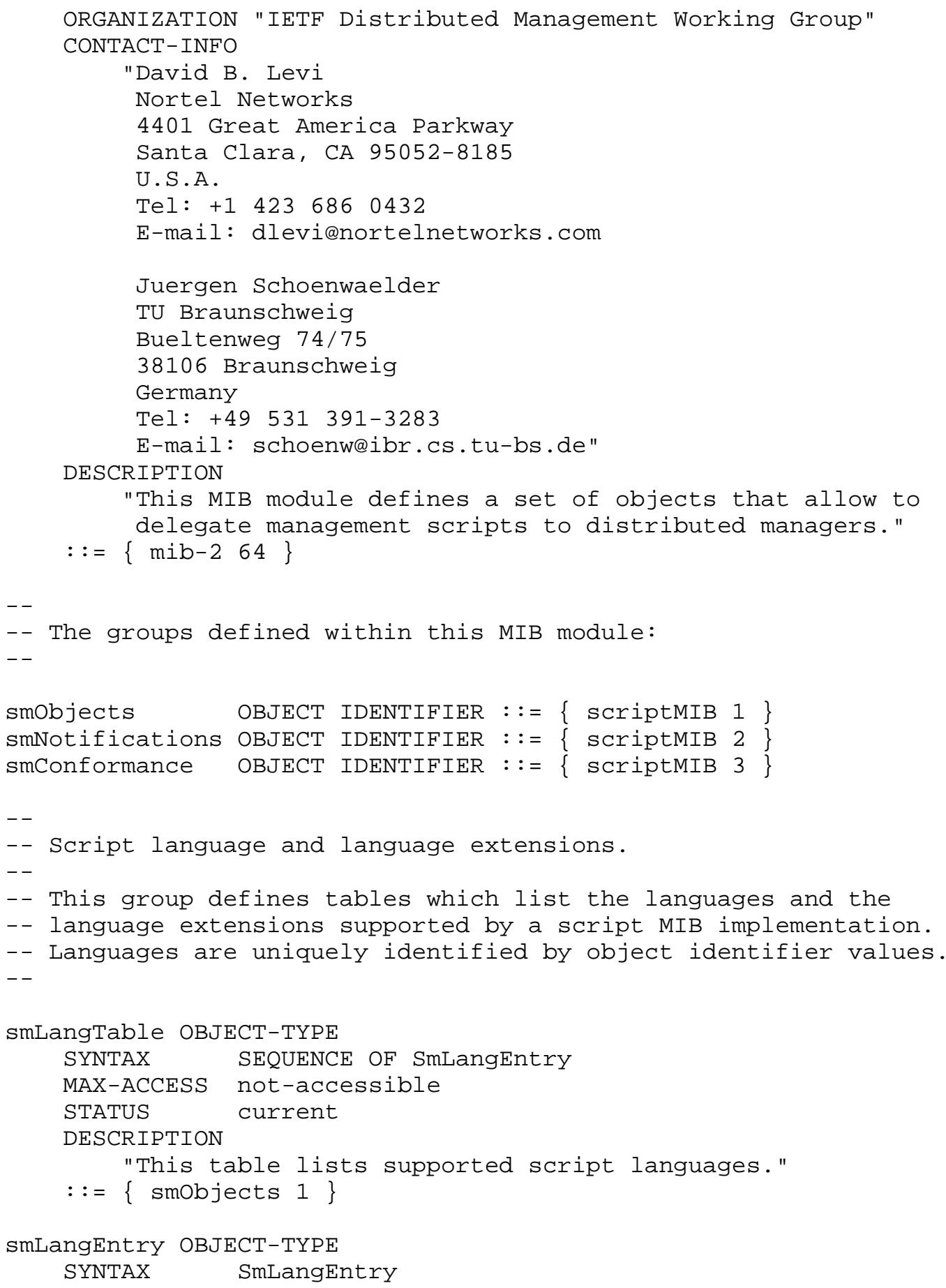




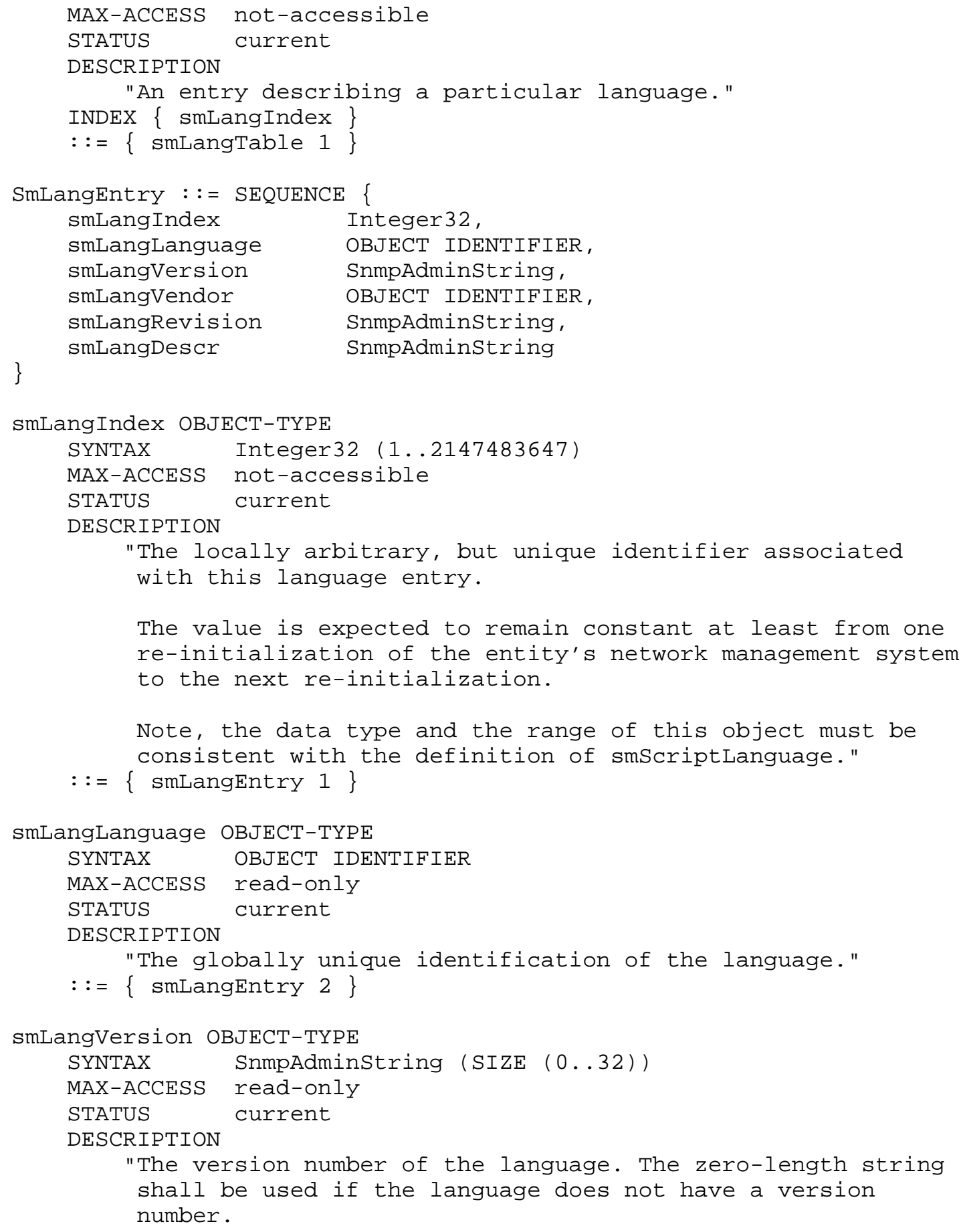




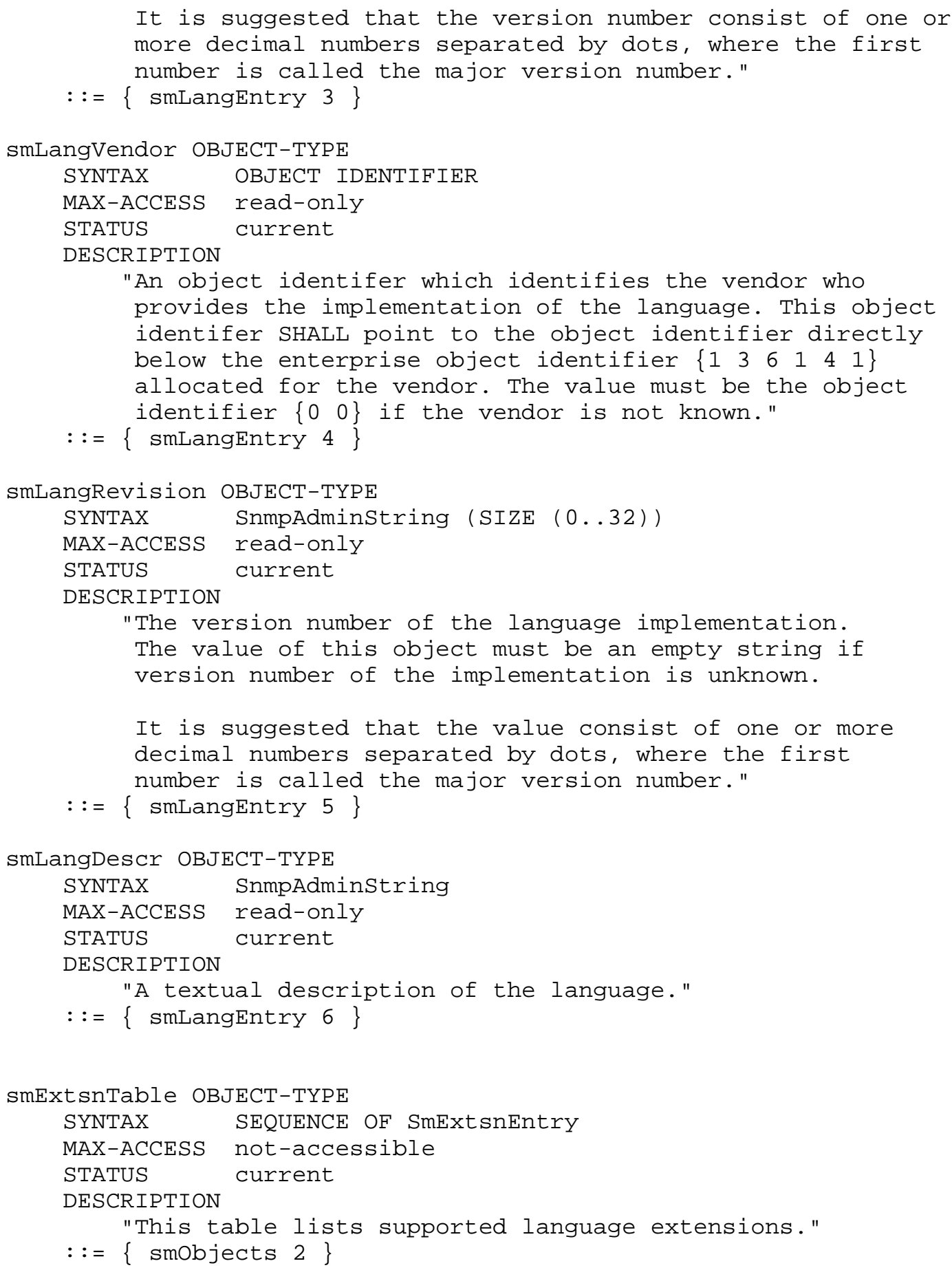




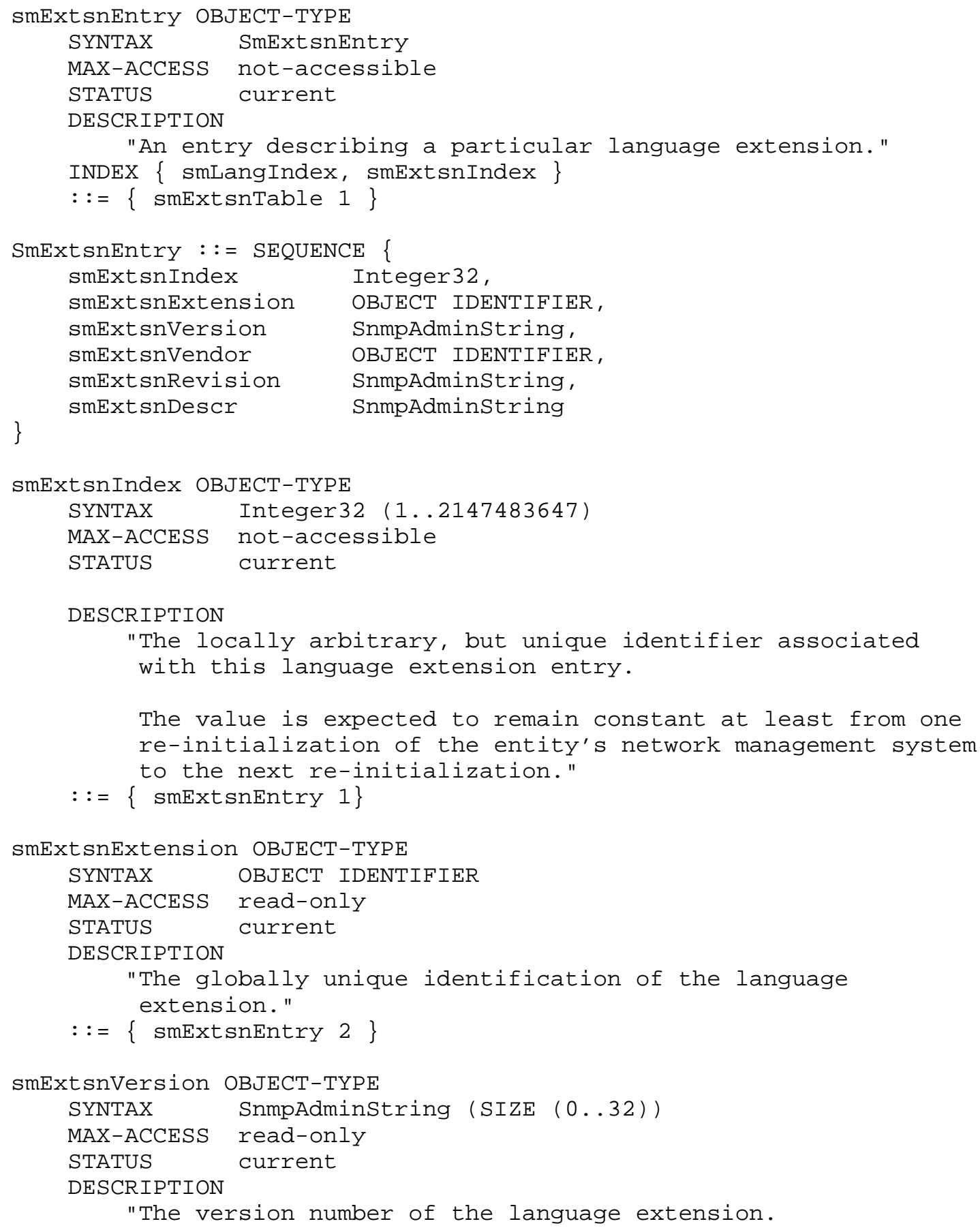

"The version number of the language extension. 


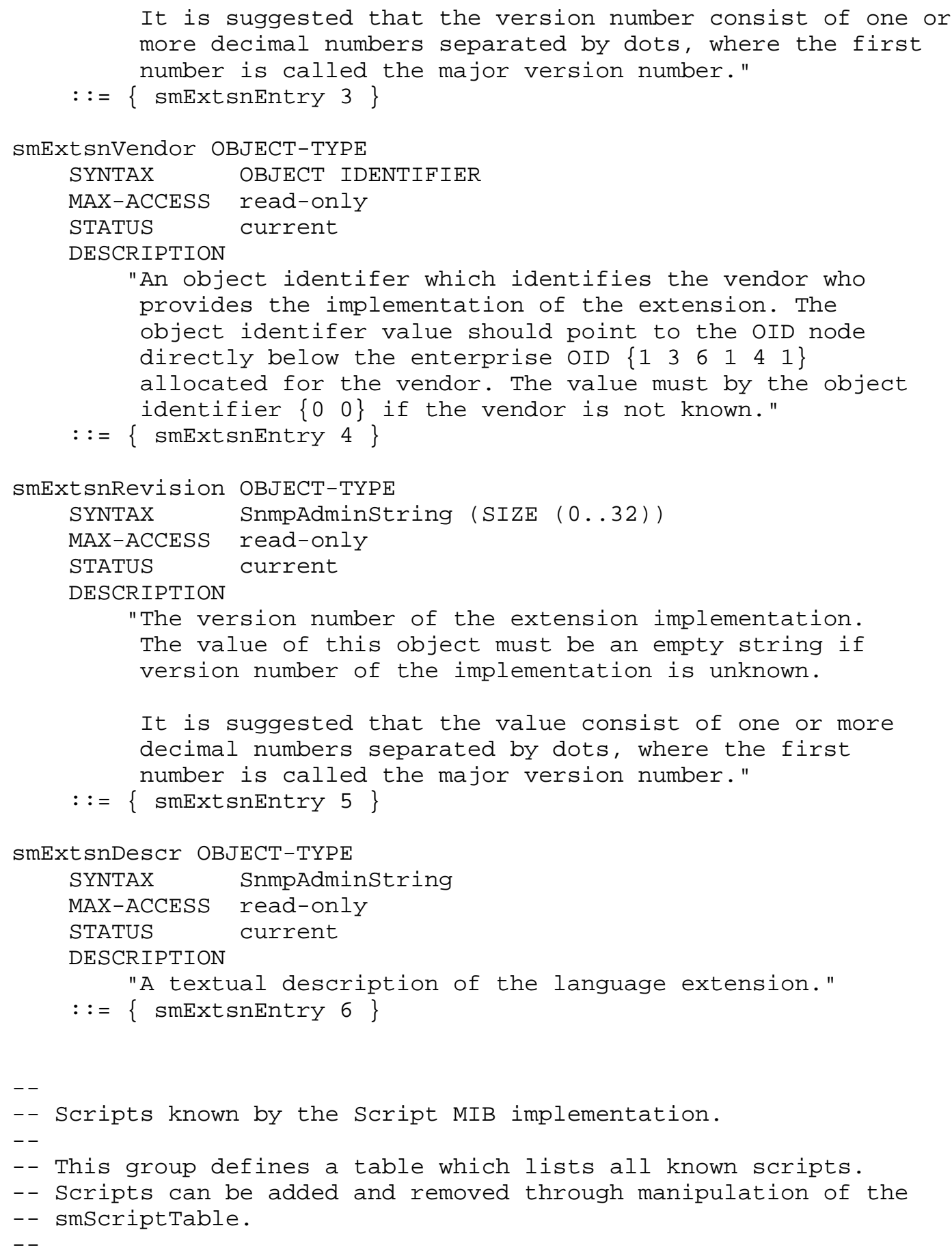




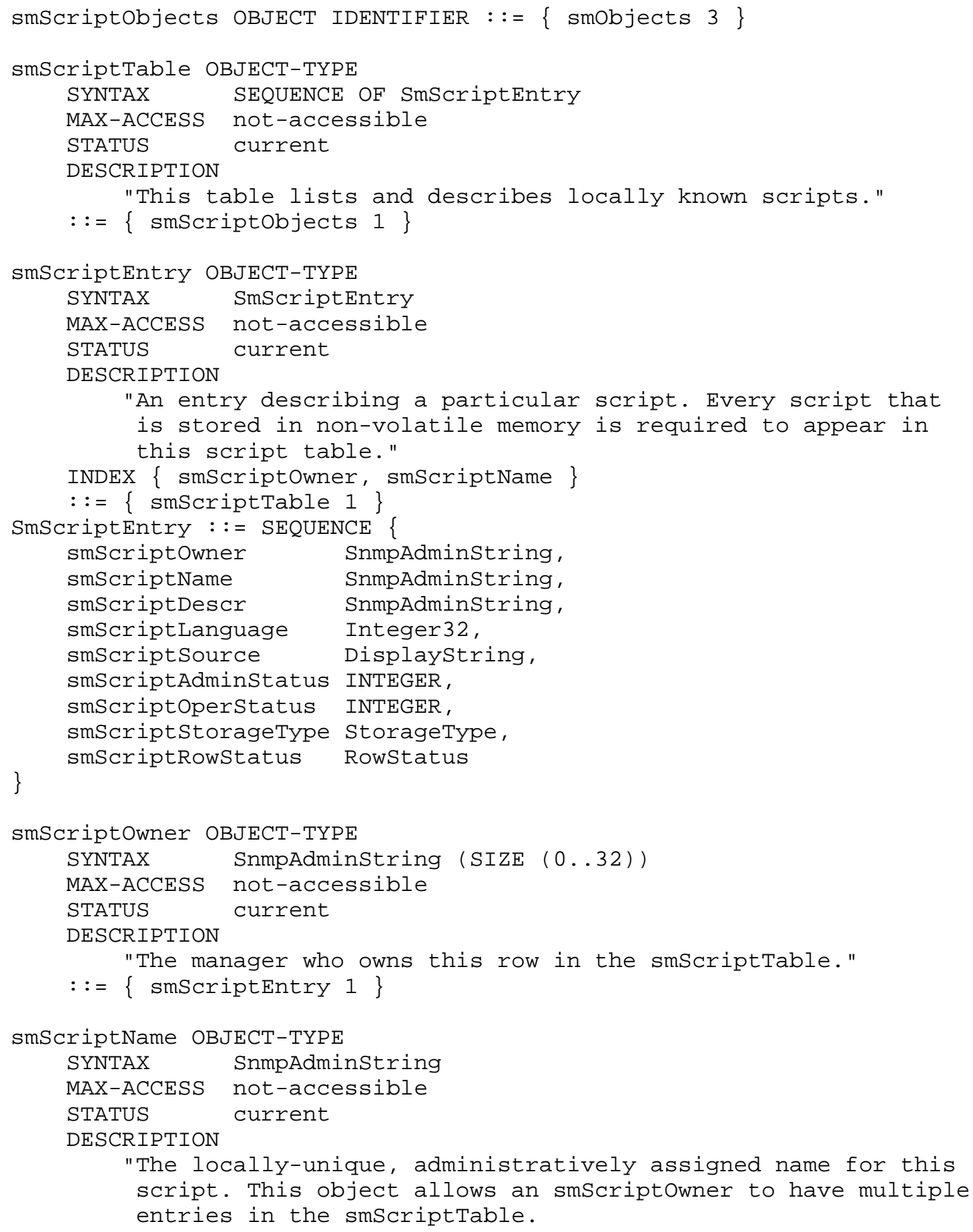




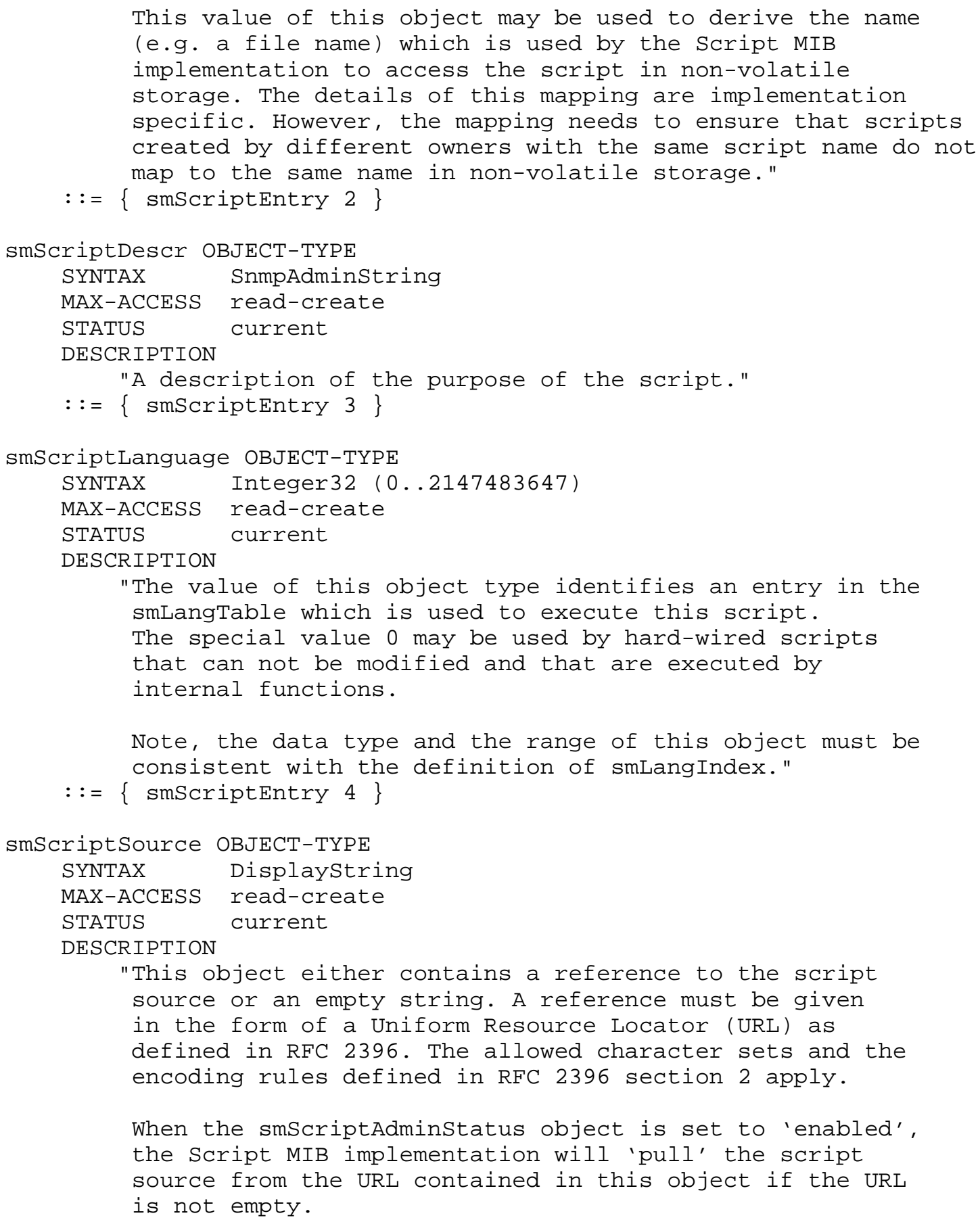




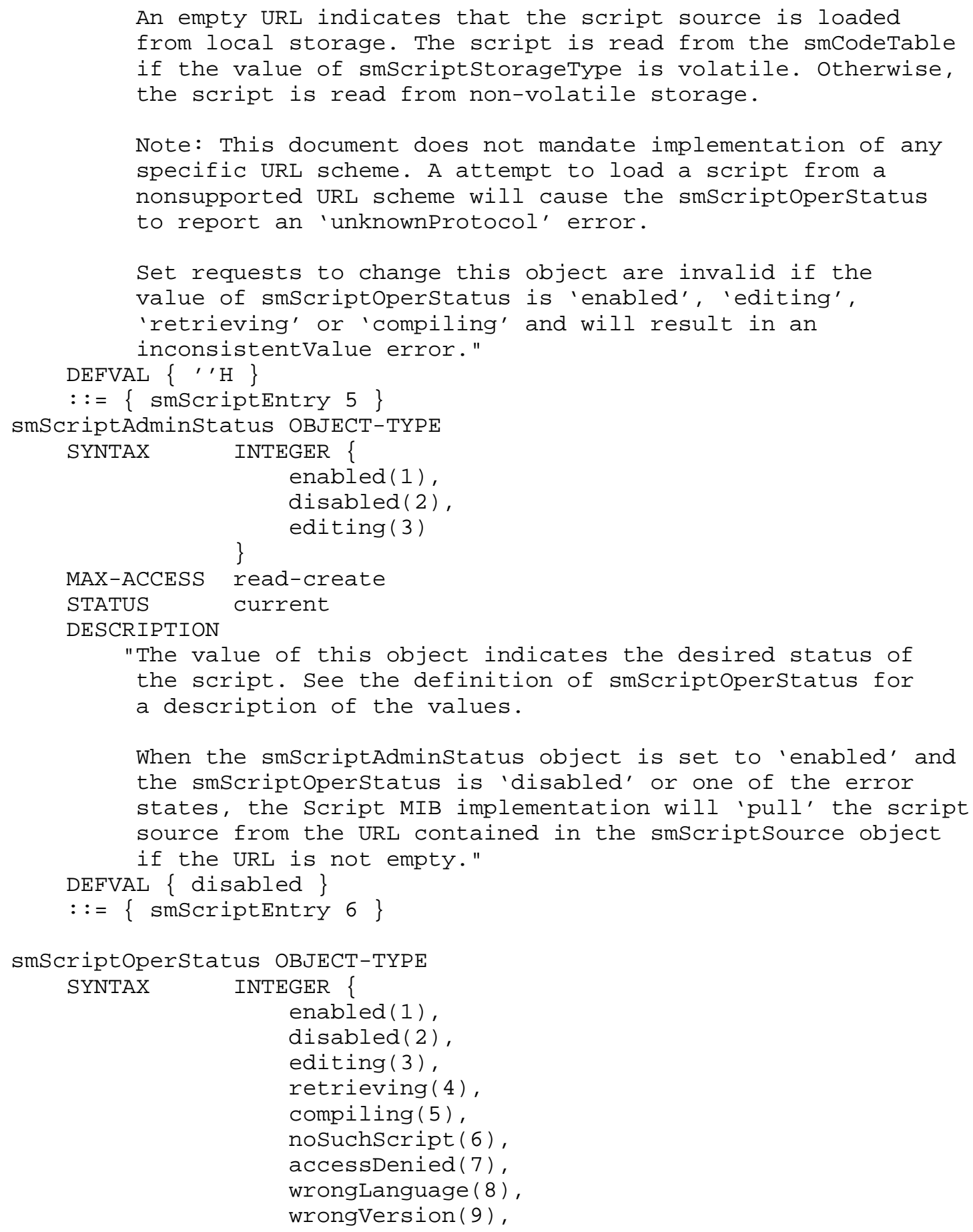




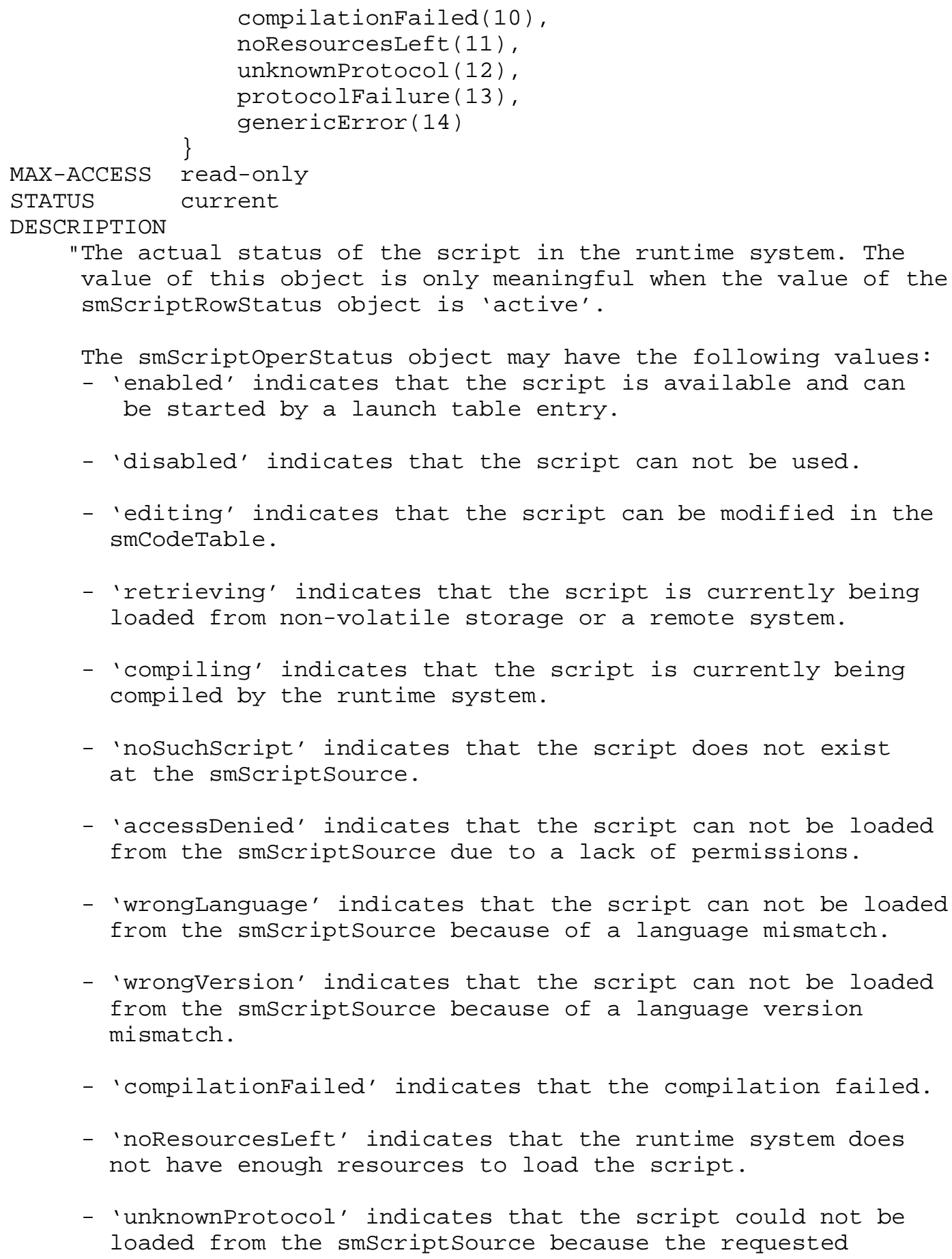




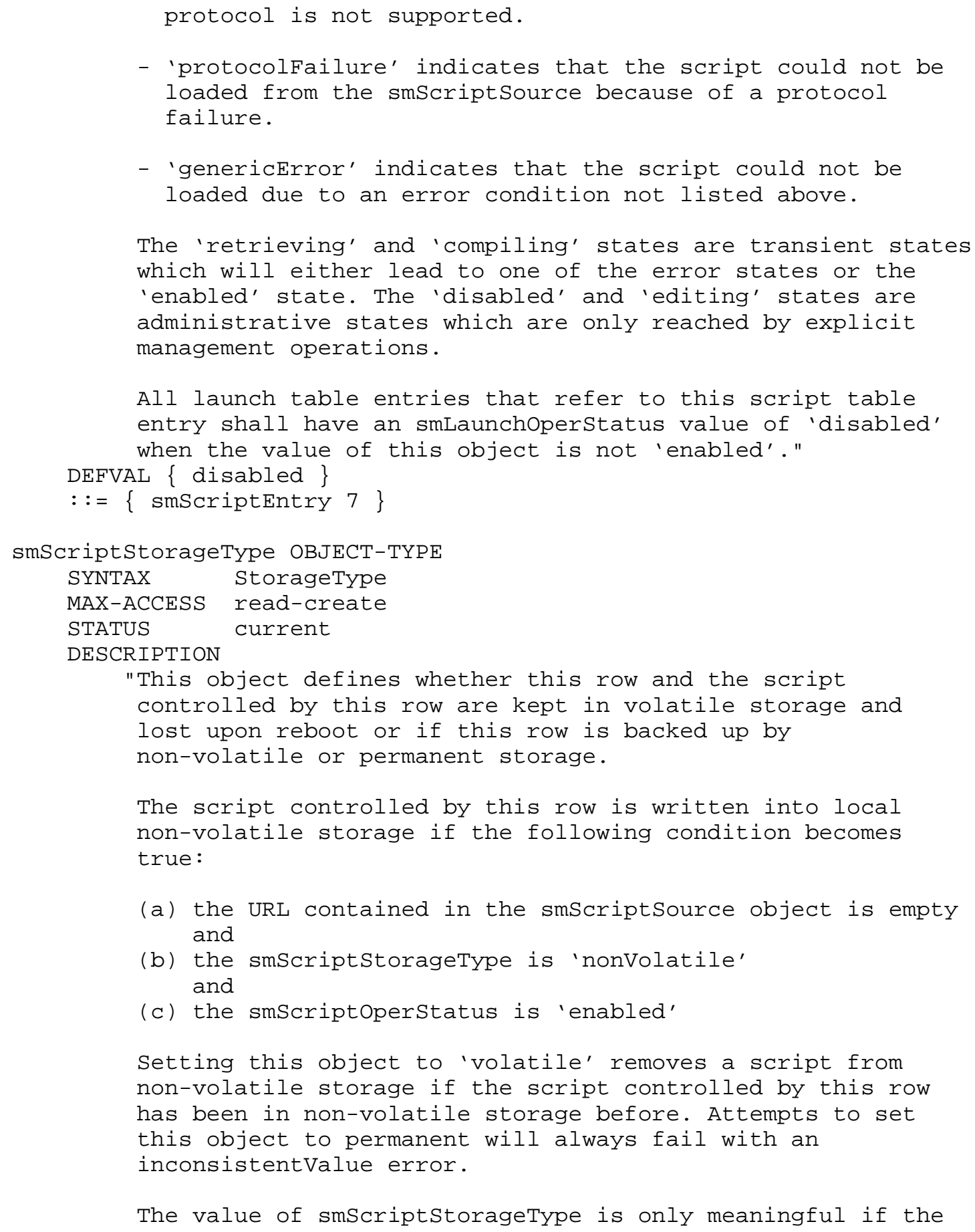

(a) the URL contained in the smScriptsource object is empty and

(b) the smScriptStorageType is 'nonVolatile' and

(c) the smScriptoperstatus is 'enabled'

Setting this object to 'volatile' removes a script from non-volatile storage if the script controlled by this row has been in non-volatile storage before. Attempts to set this object to permanent will always fail with an inconsistentvalue error.

The value of smScriptstorageType is only meaningful if the 


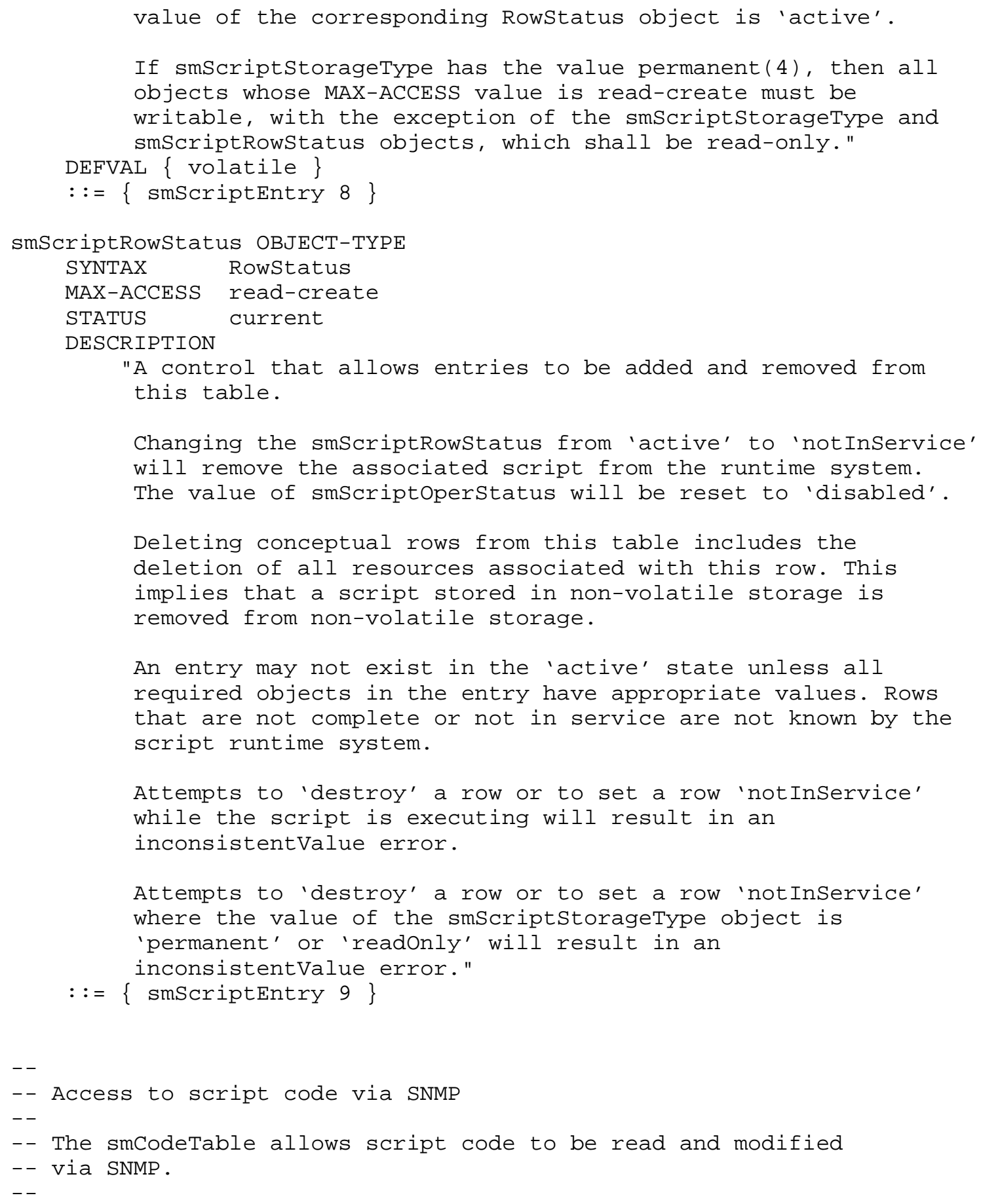




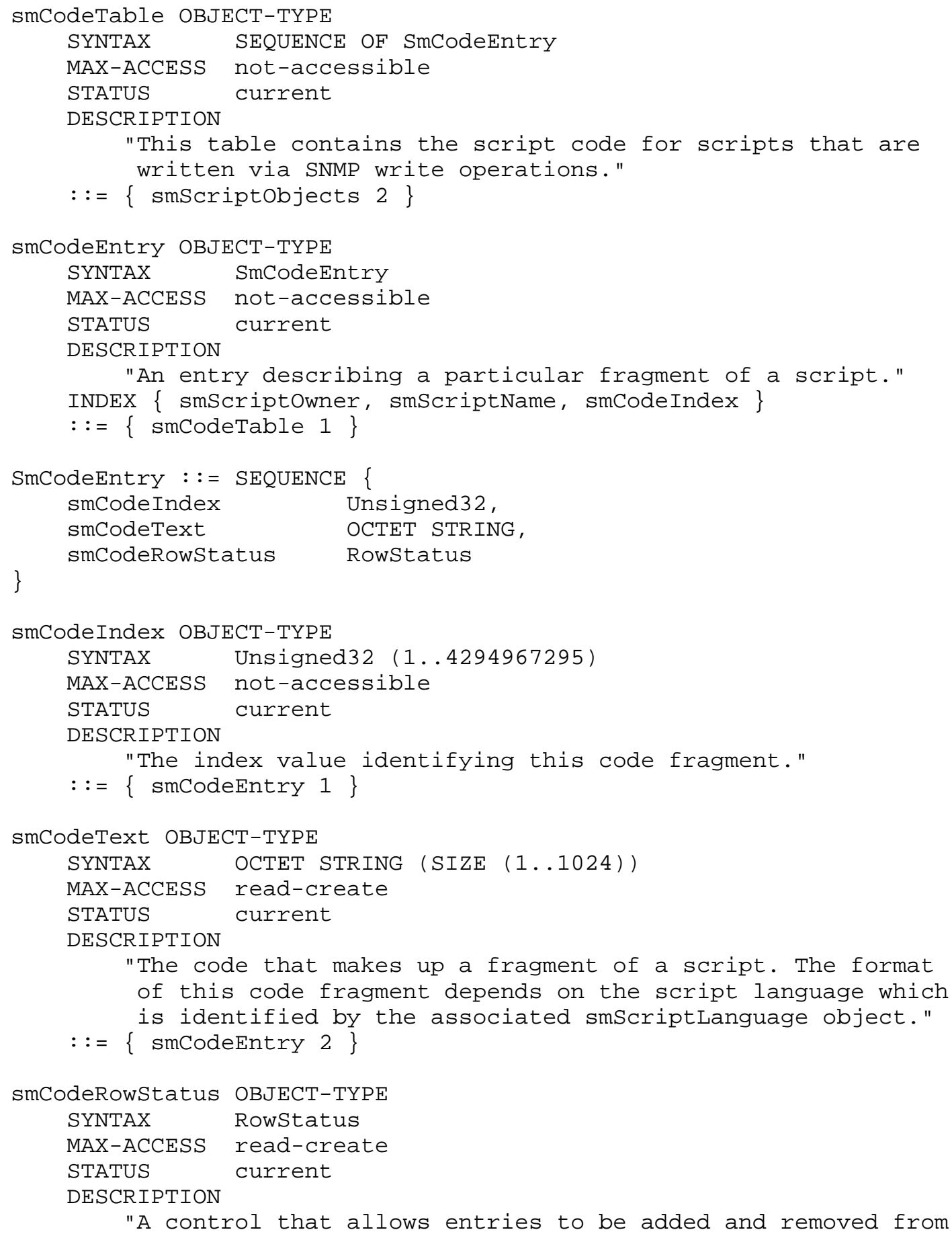

"A control that allows entries to be added and removed from 


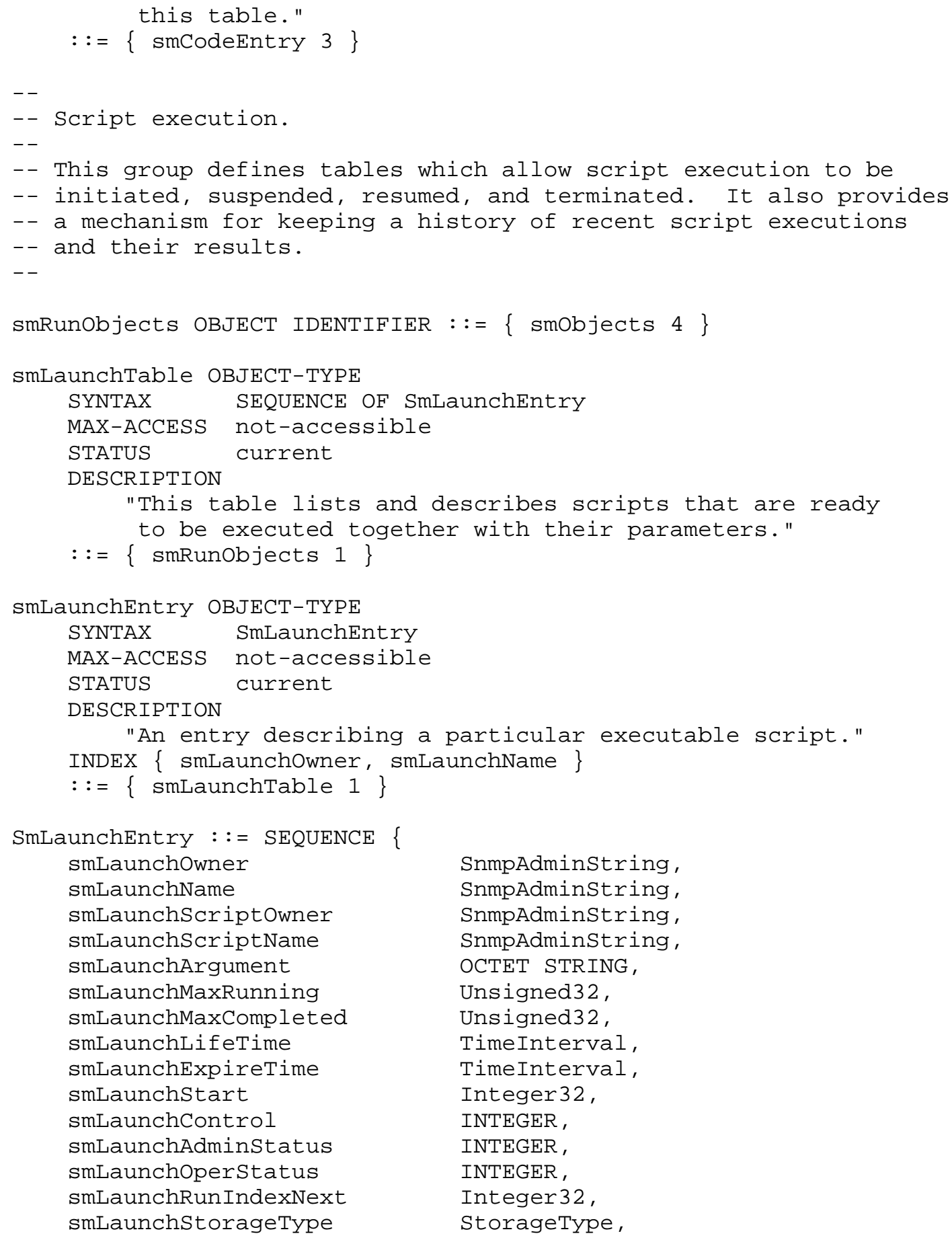




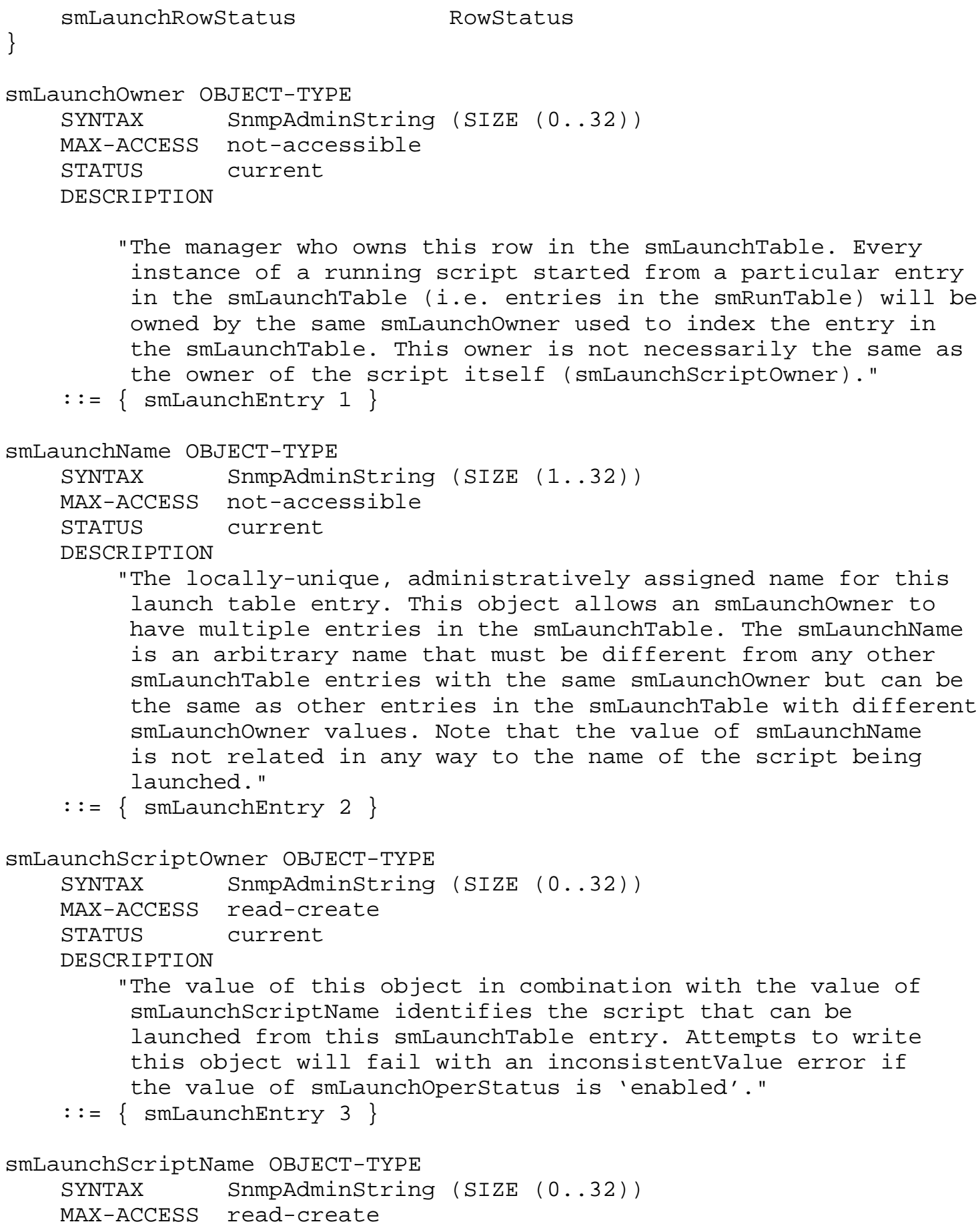




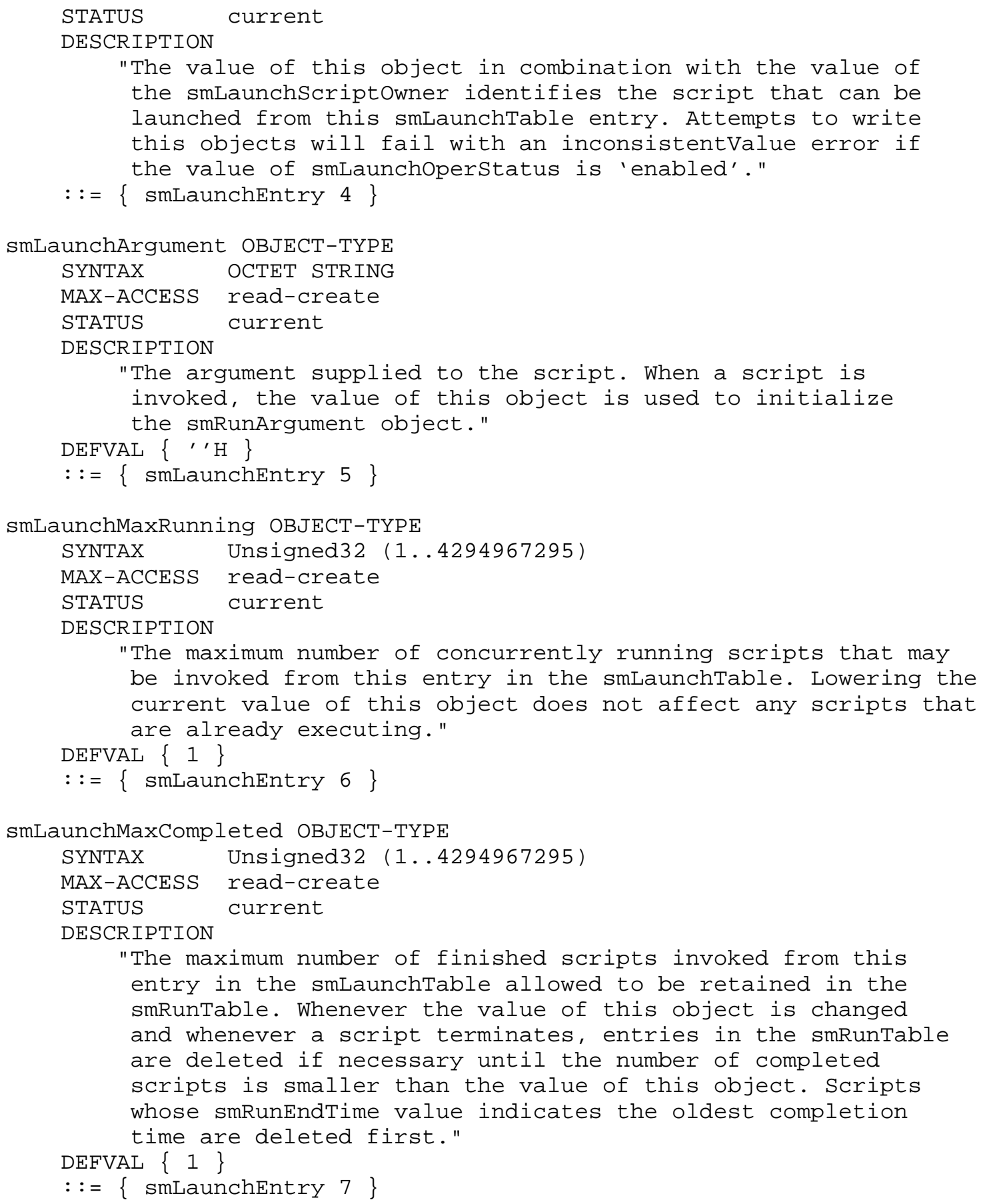




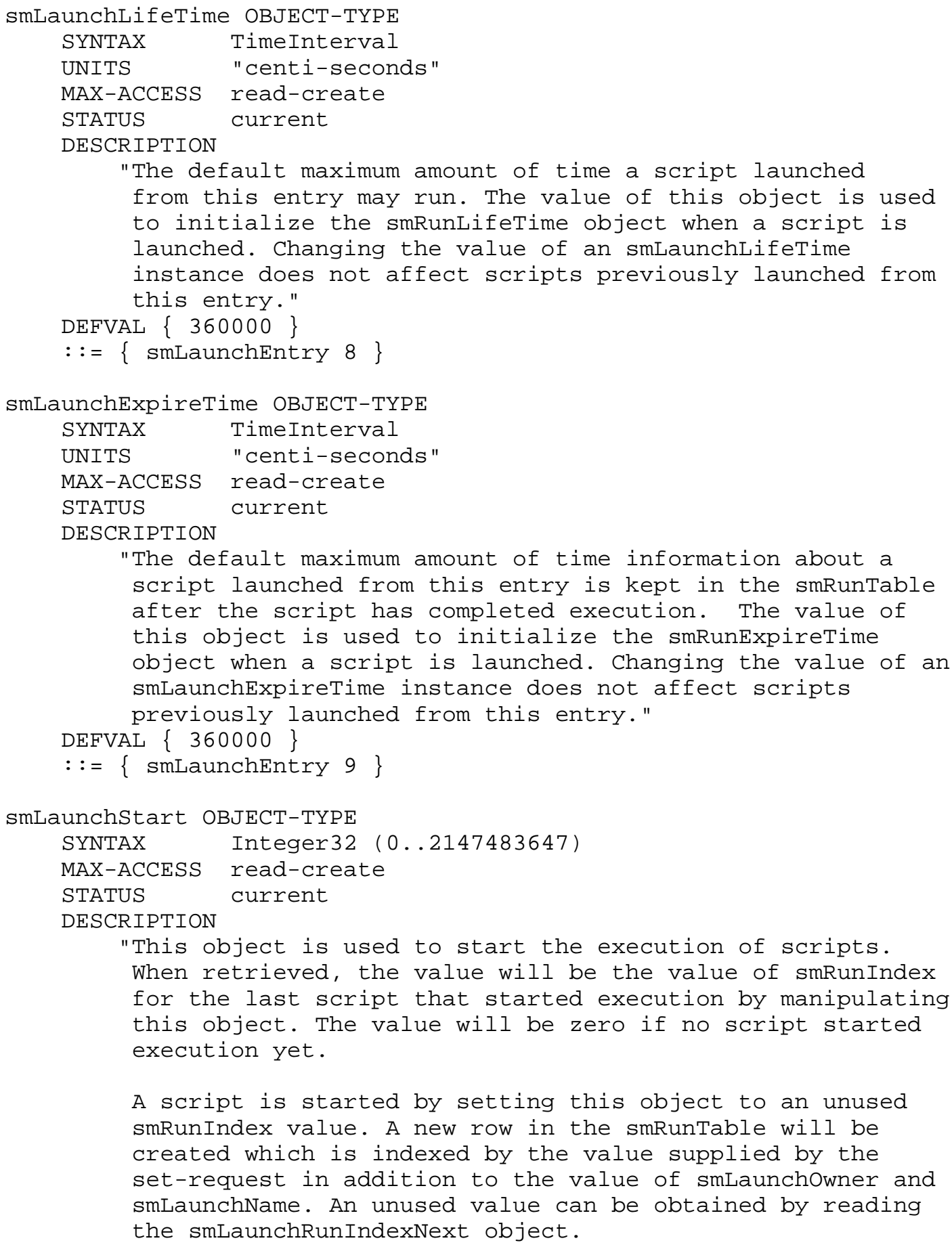


Setting this object to the special value 0 will start the script with a self-generated smRunIndex value. The consequence is that the script invoker has no reliable way to determine the smRunIndex value for this script invocation and that the invoker has therefore no way to obtain the results from this script invocation. The special value 0 is however useful for scheduled script invocations.

If this object is set, the following checks must be performed:

1) The value of the smLaunchoperstatus object in this entry of the smLaunchTable must be 'enabled'.

2) The values of smLaunchscriptowner and smLaunchscriptName of this row must identify an existing entry in the smScriptTable.

3) The value of smScriptoperstatus of this entry must be 'enabled'.

4) The principal performing the set operation must have read access to the script. This must be checked by calling the isAccessAllowed abstract service interface defined in RFC 2271 on the row in the smScriptTable identified by smLaunchScriptowner and smLaunchScriptName. The isAccessAllowed abstract service interface must be called on all columnar objects in the smScriptTable with a MAX-ACCESS value different than 'not-accessible'. The test fails as soon as a call indicates that access is not allowed.

5) If the value provided by the set operation is not 0 , a check must be made that the value is currently not in use. Otherwise, if the value provided by the set operation is 0 , a suitable unused value must be generated.

6) The number of currently executing scripts invoked from this smLaunchTable entry must be less than smLaunchMaxRunning.

Attempts to start a script will fail with an inconsistentValue error if one of the checks described above fails.

Otherwise, if all checks have been passed, a new entry in the smRunTable will be created indexed by smLaunchowner, smLaunchName and the new value for smRunIndex. The value of smLaunchArgument will be copied into smRunArgument, the value of smLaunchlifeTime will be copied to smRunLifeTime, and the value of smLaunchExpireTime 


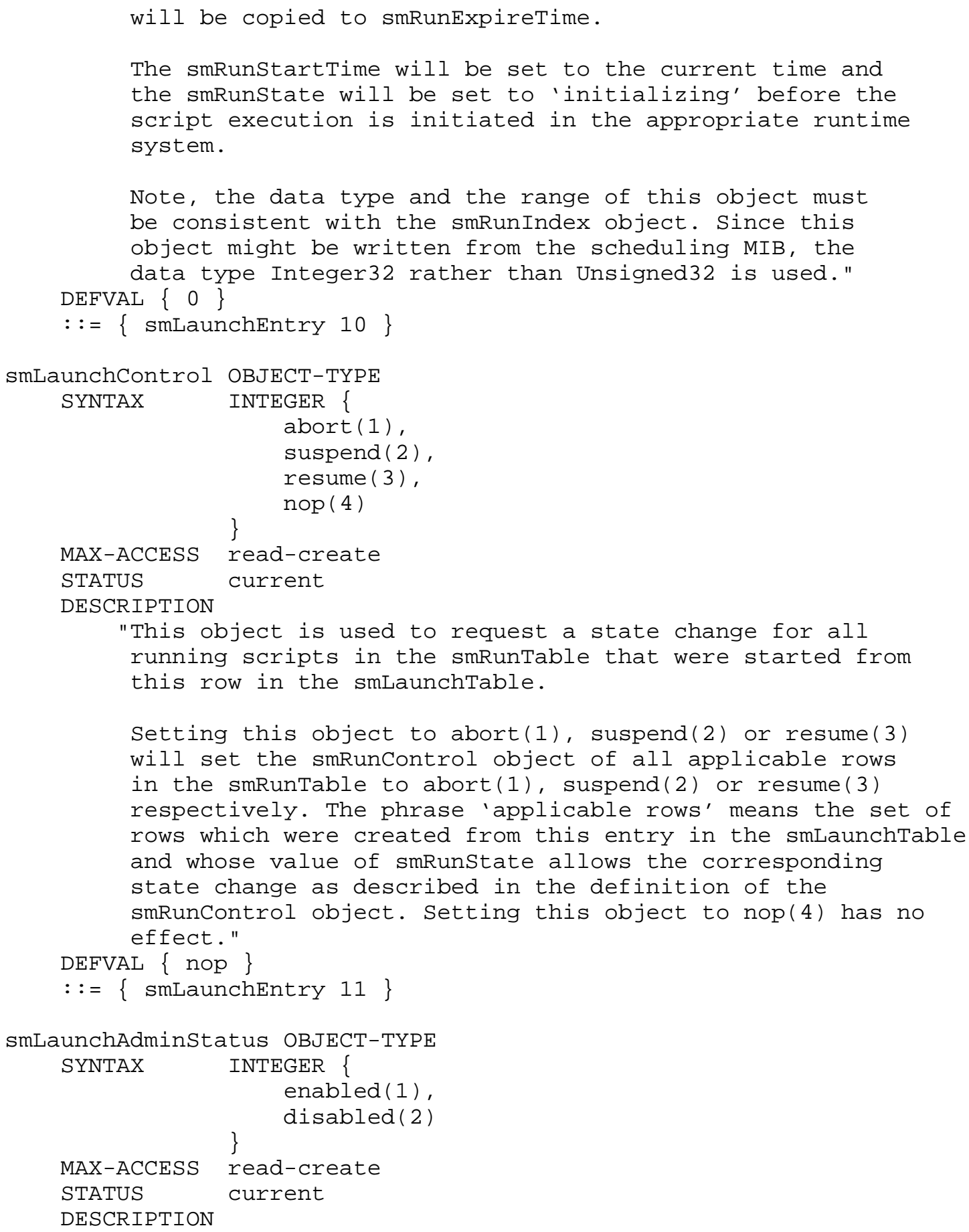




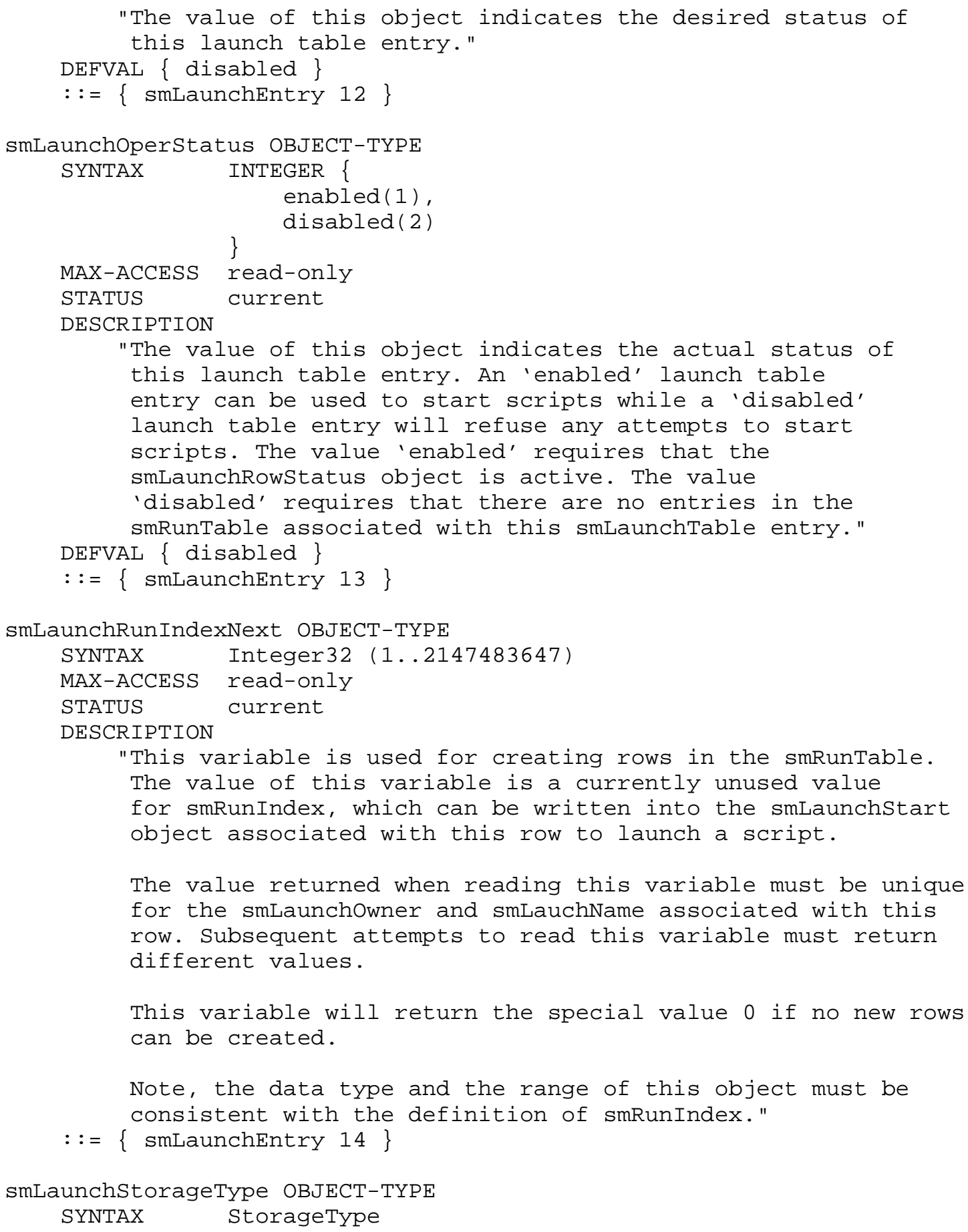




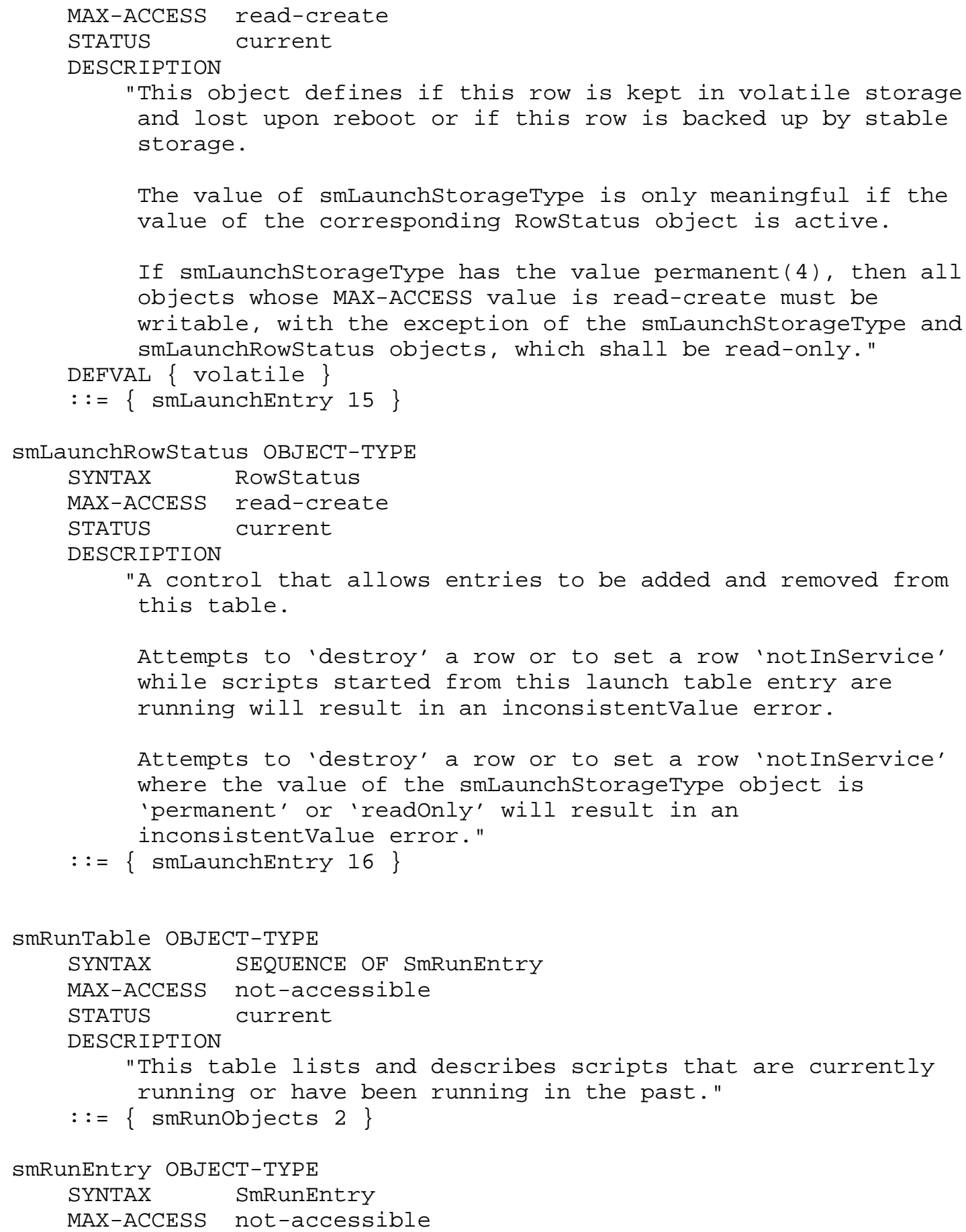




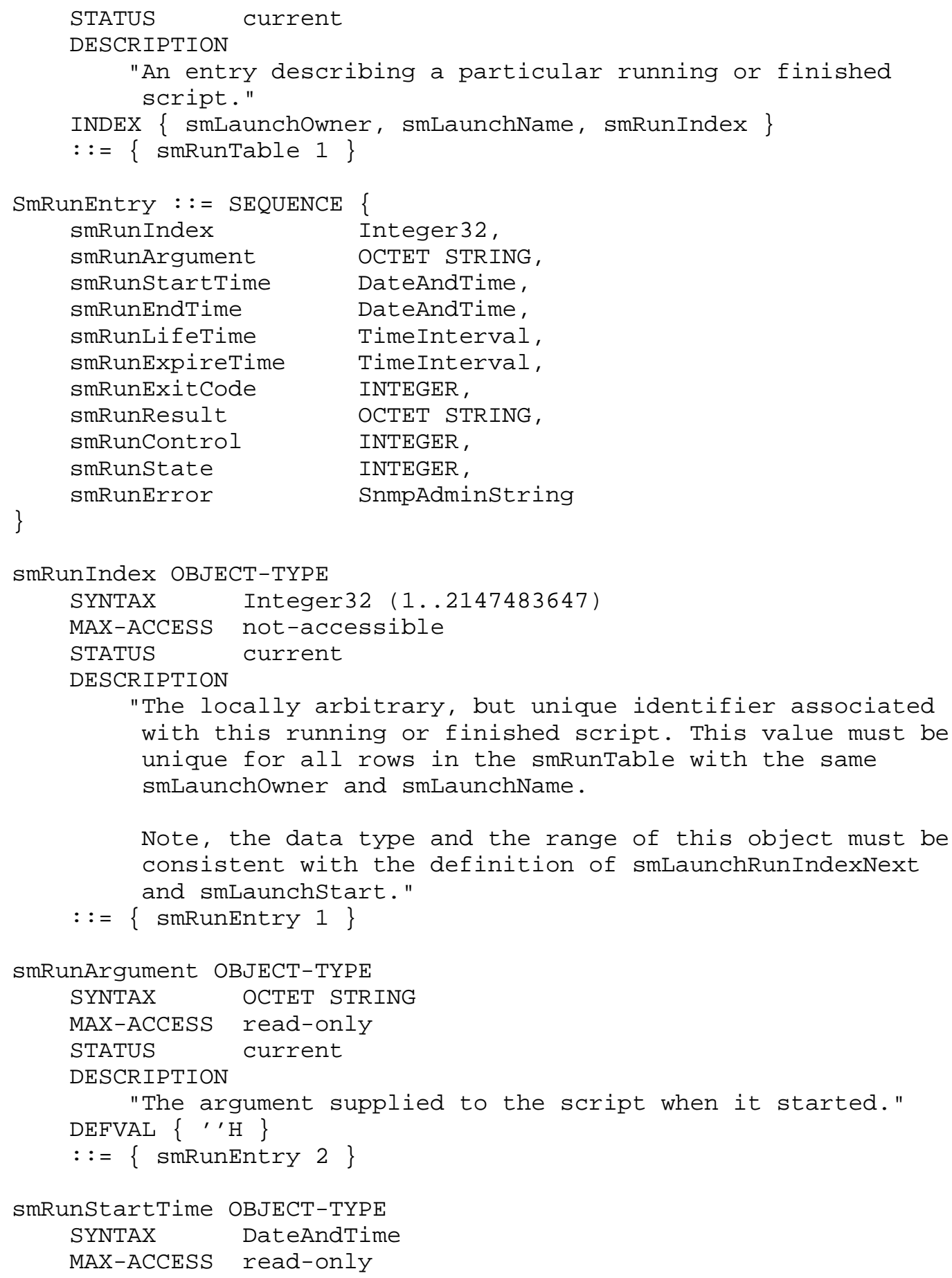




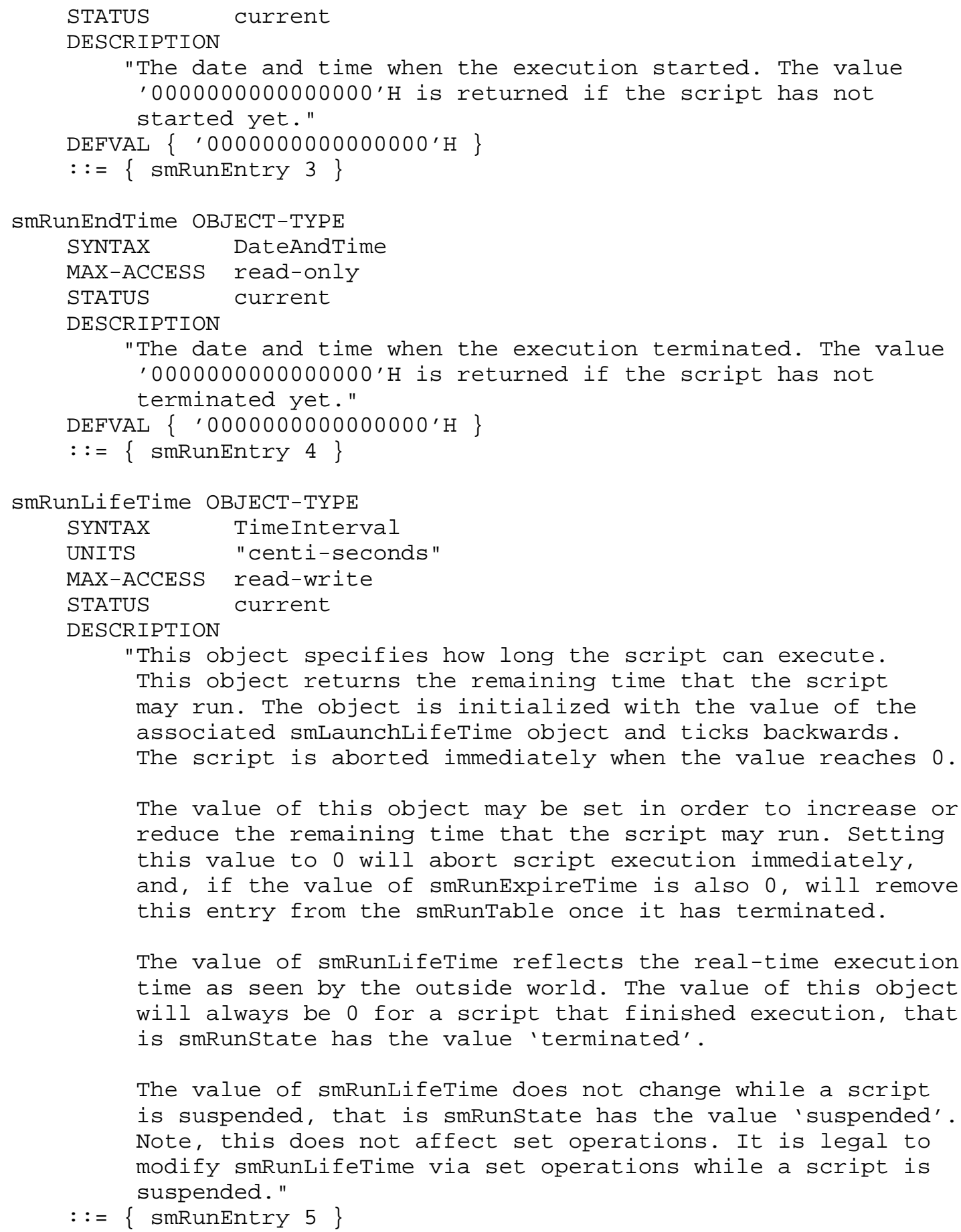




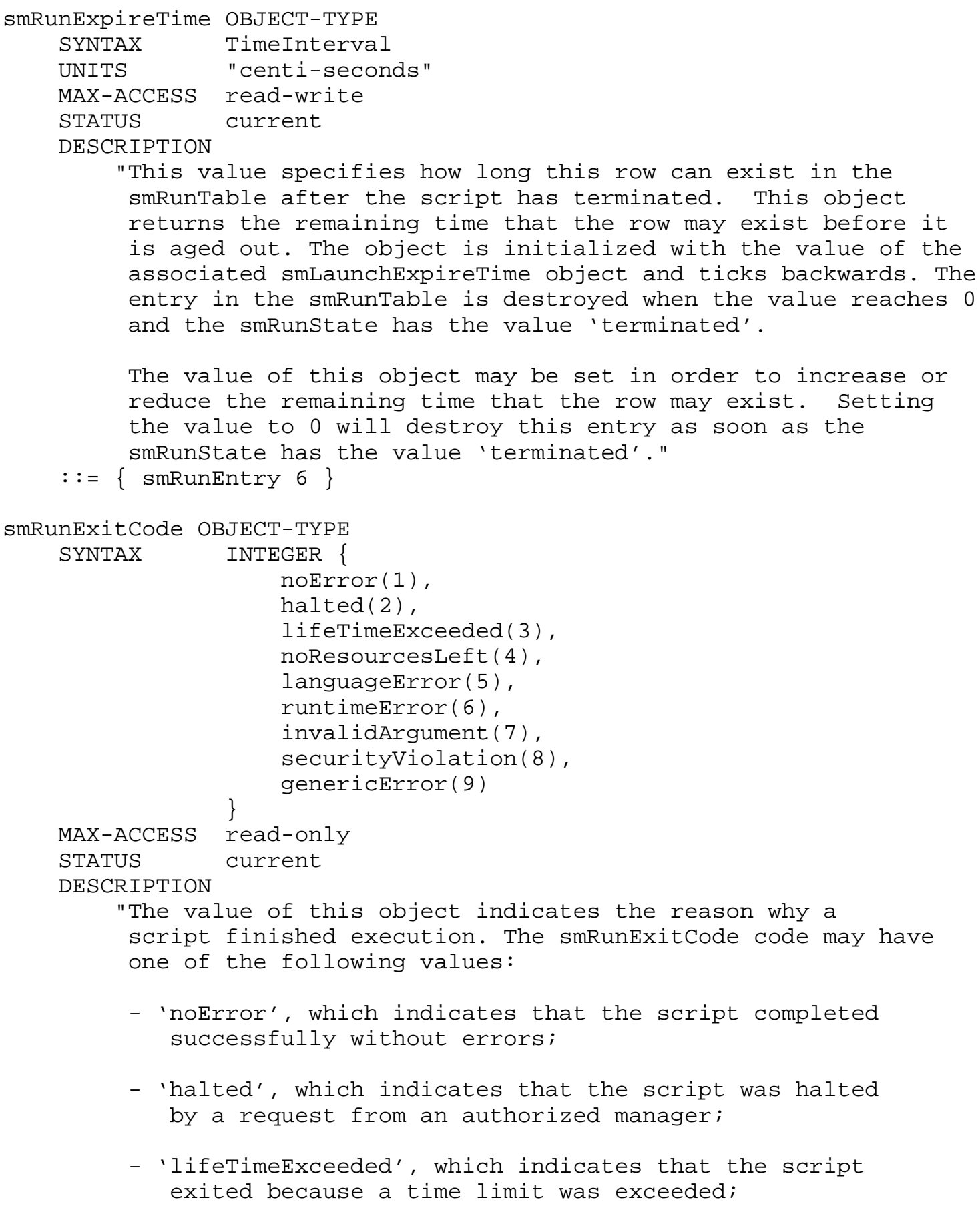




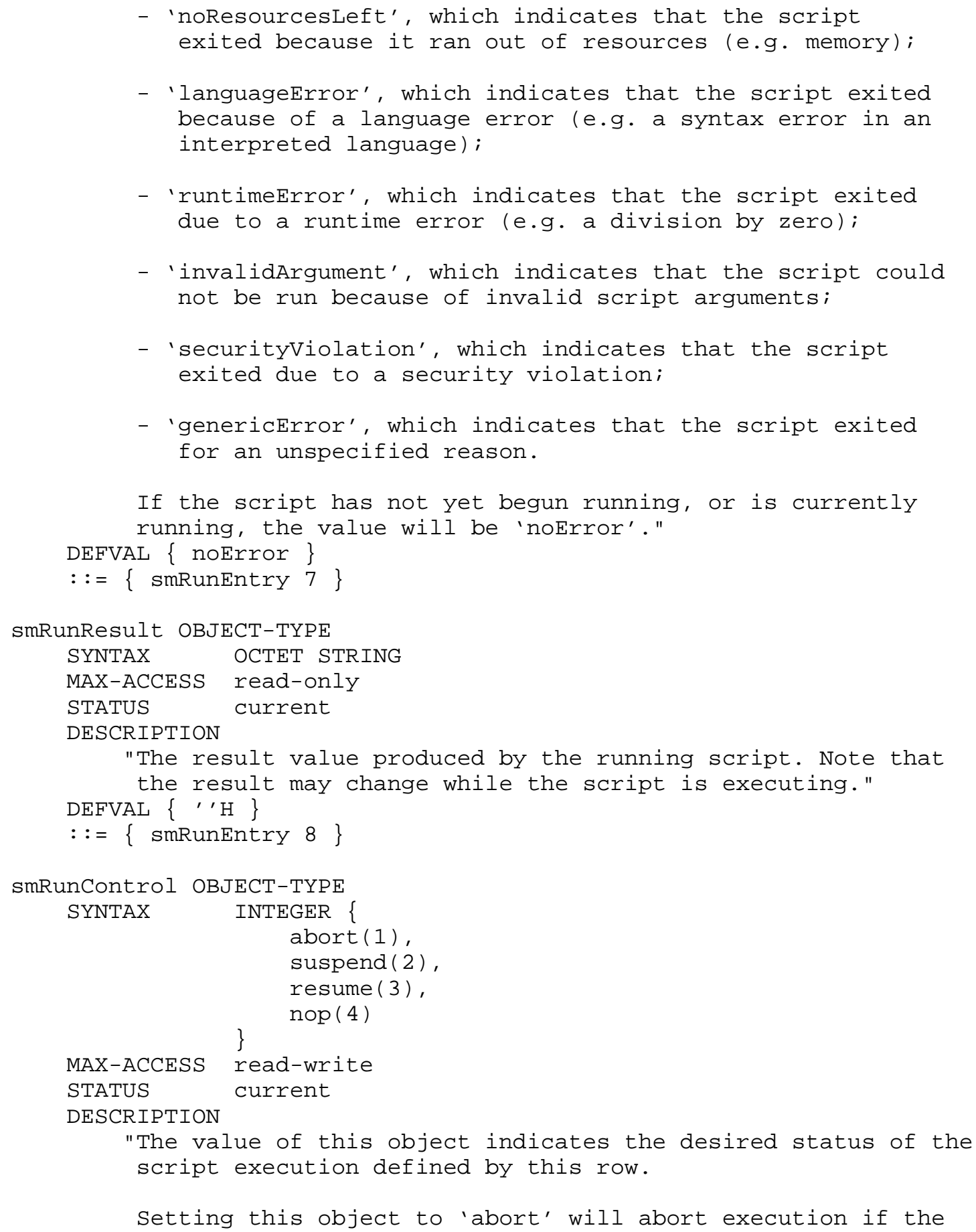




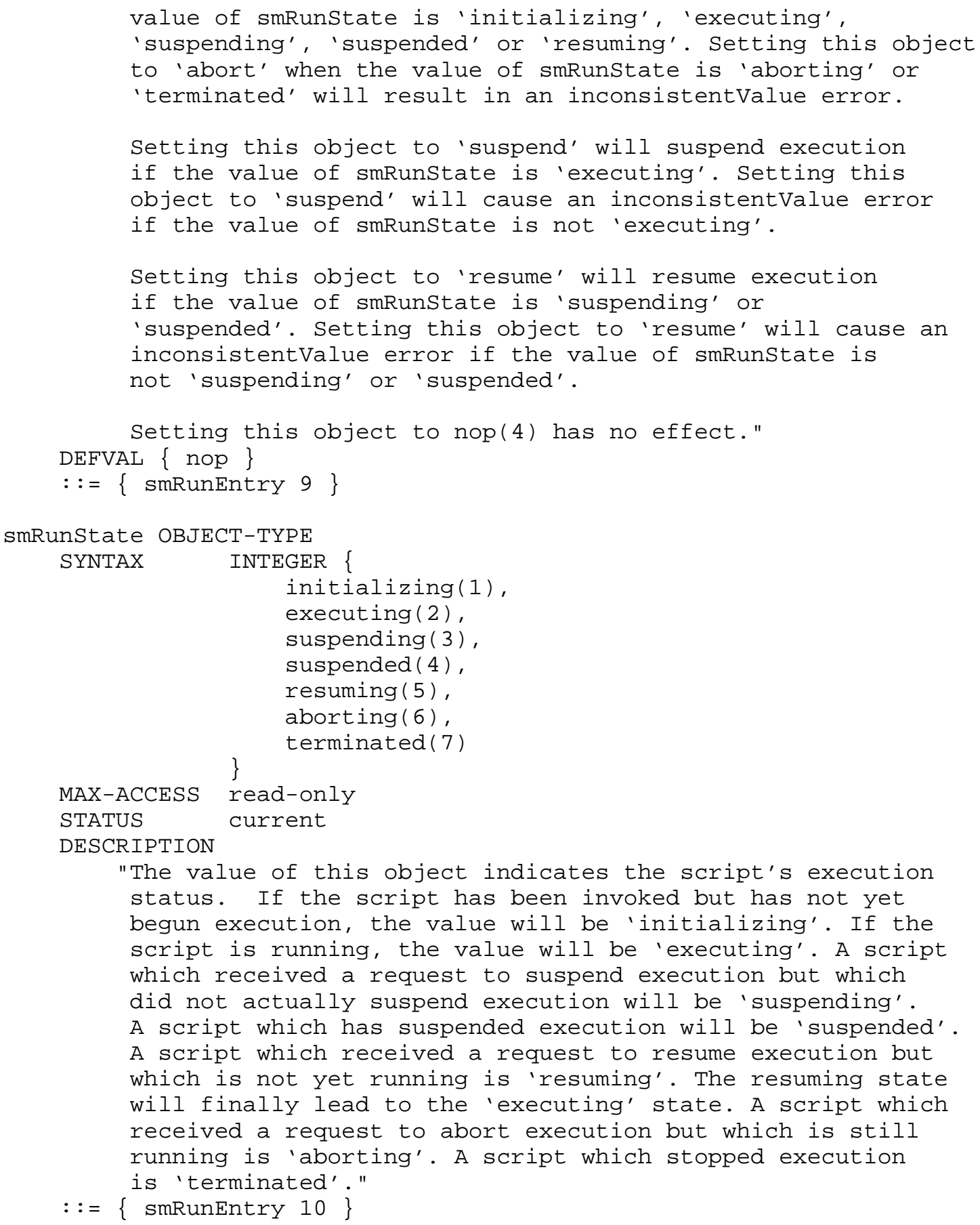




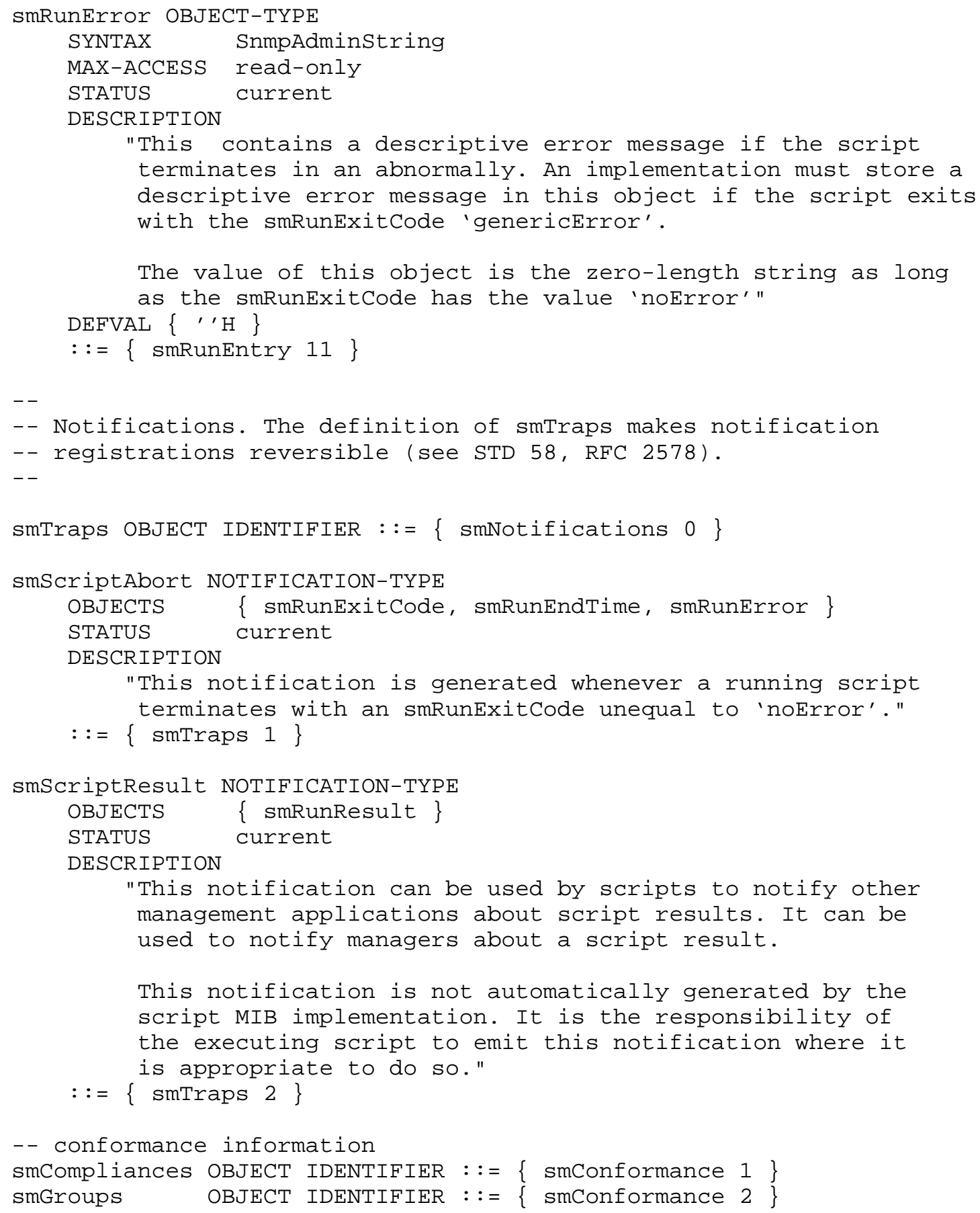




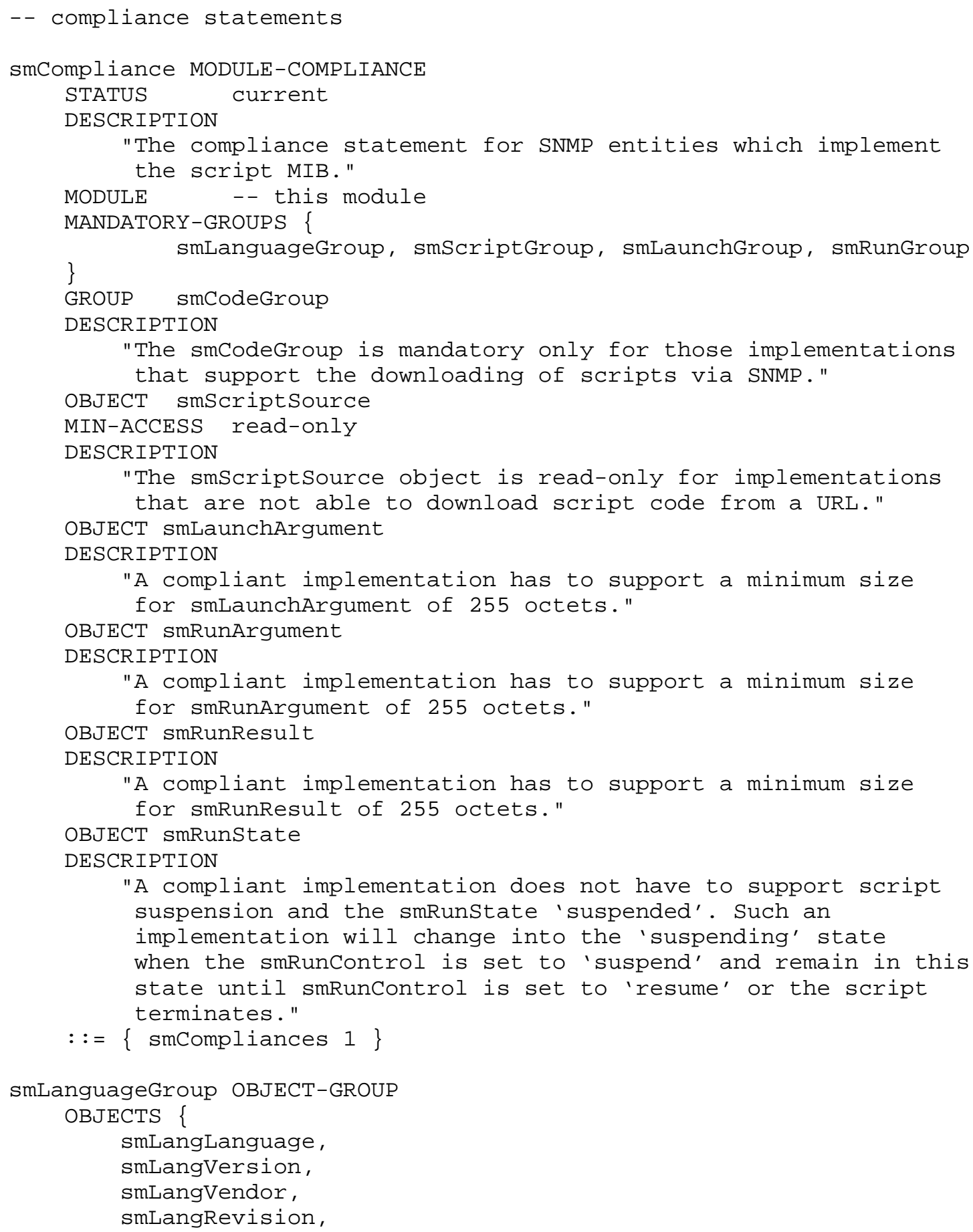




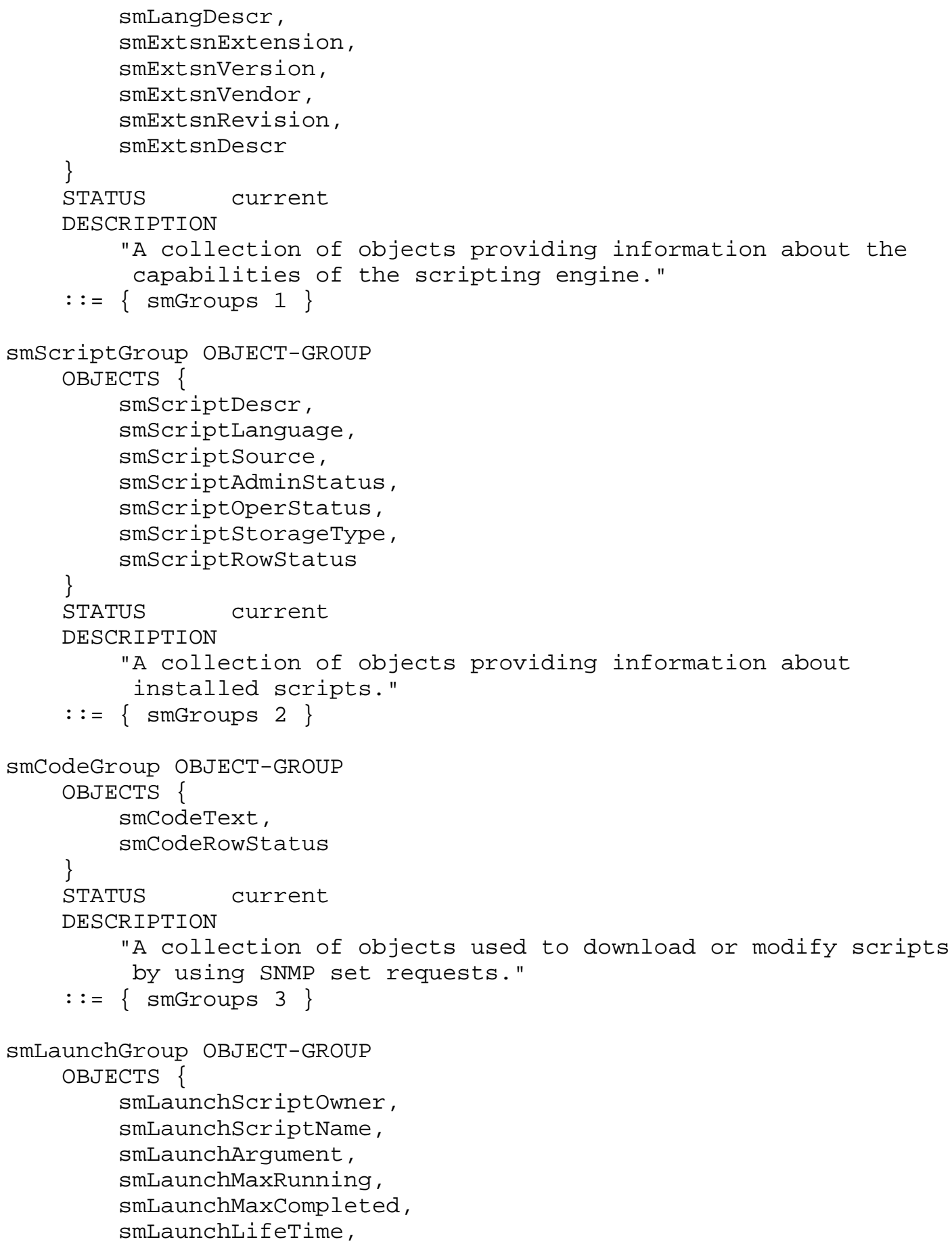




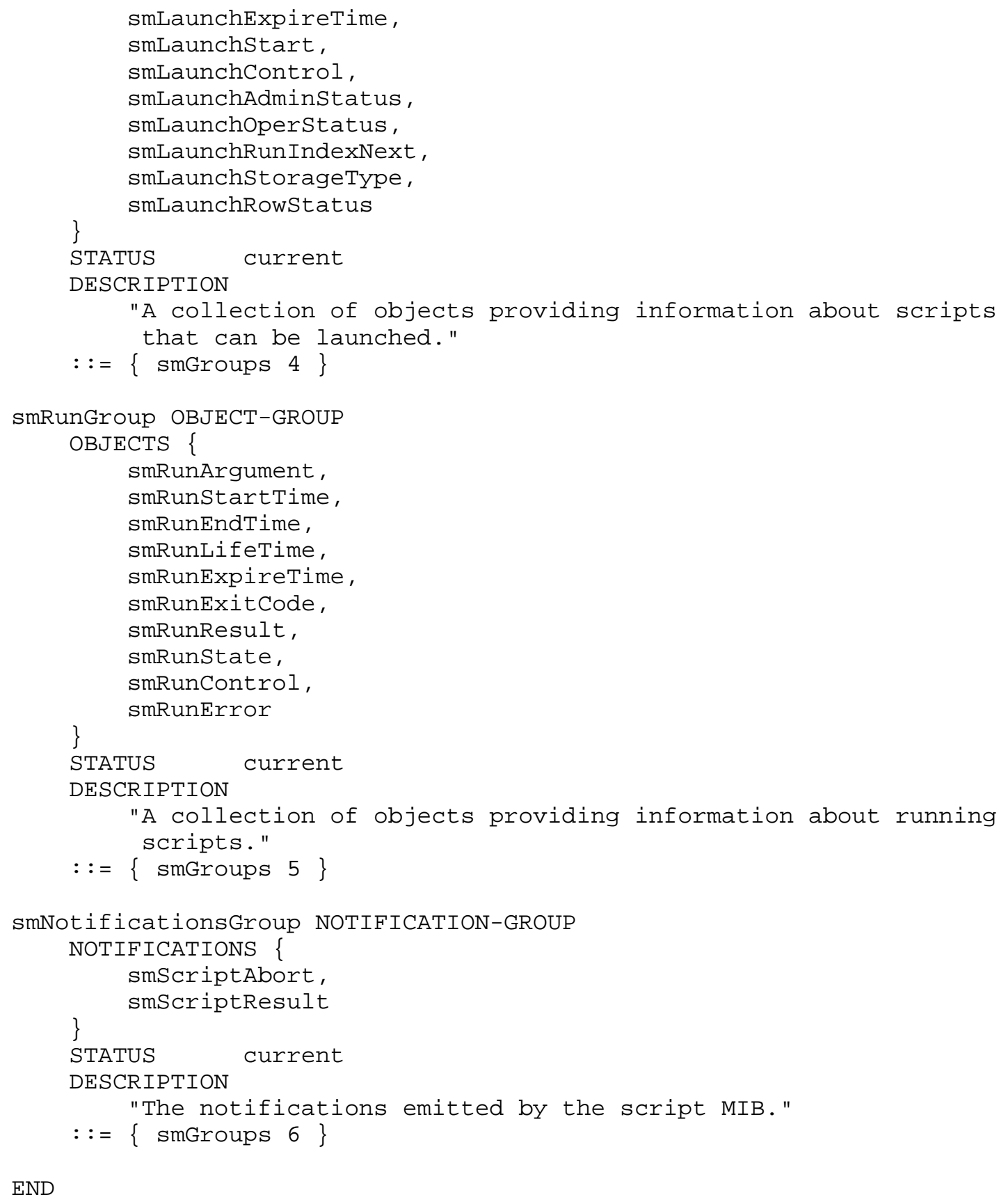

END 


\section{Usage Examples}

This section presents some examples that explain how a manager can use the Script MIB defined in this memo. The purpose of these examples is to explain the steps that are normally used to delegate management scripts.

\subsection{Pushing a script via SNMP}

This example explains the steps performed by a manager to push a script into a distributed manager.

1. The manager first checks the smLanguageTable and the smExtensionTable in order to select the appropriate script or language.

2. The manager creates a row in the smscriptTable by issuing an SNMP set-request. The smScriptRowstatus object is set to 'createAndWait' and the smScriptSource object is set to an empty string. The smScriptlanguage object is set to the language in which the script was written. The smscriptstorageType object is set to 'volatile' to indicate that the script will be loaded via the smcodeTable. The smscriptowner is set to a string which identifies the principal who owns the new row. The smScriptName defines the administratively assigned unique name for the script.

3. The manager sets the smScriptRowstatus object to 'active' and the smScriptAdminstatus object to 'editing'.

4. The manager pushes the script to the distributed manager by issuing a couple of SNMP set-requests to fill the smcodeTable.

5. Once the whole script has been transferred, the manager sends a set-request to set the smScriptAdminStatus object to 'enabled'. The Script MIB implementation now makes the script accessible to the runtime system. This might include the compilation of the script if the language requires a compilation step.

6. The manager polls the smScriptoperstatus object until the value is either 'enabled' or one of the error status codes. The script can only be used if the value of smScriptoperstatus is 'enabled'.

7. If the manager wants to store the script in local non-volatile storage, it should send a set-request which changes the smScriptStorageType object to 'nonVolatile'. 


\subsection{Pulling a script from a URL}

This example explains the steps performed by a manager to cause a distributed manager to pull a script from a URL.

1. The manager first checks the smLanguageTable and the smExtensionTable in order to select the appropriate script or language.

2. The manager creates a row in the smscriptTable by issuing an SNMP set-request. The smScriptRowstatus object is set to 'createAndWait' and the smScriptSource object is set to the URL which points to the script source. The smScriptlanguage object is set to the language in which the script was written. The smscriptowner is set to a string which identifies the principal who owns the new row. The smScriptName defines the administratively assigned unique name for the script.

3. The manager sets the smScriptRowstatus object to 'active'.

4. The manager sends a set-request to set the smScriptAdminstatus object to 'enabled'. The Script MIB implementation now makes the script accessible to the runtime system. This causes a retrieval operation to pull the script from the URL stored in smScriptsource. This retrieval operation might be followed by a compile operation if the language requires a compilation step.

5. The manager polls the smScriptoperstatus object until the value is either 'enabled' or one of the error status codes. The script can only be used if the value of smScriptoperstatus is 'enabled'.

6. If the manager wants to store the script in local non-volatile storage, it should send a set-request which changes the smScriptstorageType object to 'nonVolatile'.

7.3. Modifying an existing script

This section explains how a manager can modify a script by sending

SNMP set-requests.

1. First, the script is de-activated by setting the smScriptAdminstatus to 'disabled'.

2. The manager polls the smScriptoperstatus object until the value is 'disabled'. 
3. The manager sets smScriptsource to an empty string and smScriptAdminstatus to 'editing'. This makes the script source available in the smcodeTable.

4. The manager polls the smScriptoperStatus object until the value is 'editing'.

5. The manager sends SNMP set-requests to modify the script in the smCodeTable.

6. The manager sends a set-request to set the smScriptAdminstatus object to 'enabled'. The Script MIB implementation now makes the script accessible to the runtime system. This might include the compilation of the script if the language requires a compilation step.

7. The manager polls the smScriptoperStatus object until the value is either 'enabled' or one of the error status codes. The script can only be used if the value of smScriptoperstatus is 'enabled'.

7.4. Removing an existing script

This section explains how a manager can remove a script from a distributed manager.

1. First, the manager sets the smScriptAdminStatus to 'disabled'. This will ensure that no new scripts can be started while running scripts finish their execution.

2. The manager polls the smScriptoperStatus object until the value is 'disabled'.

3. The manager sends an SNMP set-request to change the smScriptRowstatus object to 'destroy'. This will remove the row and all associated resources from the Script MIB implementation.

7.5. Creating a launch button

This section explains how a manager can create a launch button for starting a script.

1. The manager, who is identified by an smLaunchowner value, first chooses a name for the new row in the smLaunchiable. The manager sends an SNMP set-request to set the smLaunchRowStatus object for this smLaunchowner and smLaunchName to 'createAndWait'. 
2. The manager fills the new smLaunchtable row with all required parameters. The smLaunchscriptowner and smLaunchScriptName values point to the script that should be started from this launch button.

3. The manager sends a set-request to change smLaunchAdminstatus to 'enabled' once the new smLaunchiable row is complete.

4. The manager polls the smLaunchoperstatus object until the value is 'enabled'.

7.6. Launching a script

This section explains the suggested way to launch a script from a given launch button.

1. The manager first retrieves the value of smLaunchRunIndexNext from the launch button selected to start the script.

2. The manager sends an SNMP set-request to set the smLaunchstart object to the value obtained in step 1 . This will launch the script if all necessary pre-conditions are satisfied (see the definition of smlaunchstart for more details). The manager can also provide the smLaunchArgument in the same set-request that is used to start the script. Upon successful start, a new row will be created in the smRunTable indexed by smLaunchowner, smLaunchName and the value written to smLaunchstart.

Note, the first step is not required. A manager can also try to guess an unused value for smRunIndex if he wants to start script in a single transaction. A manager can also use the special value 0 if he does not care about the results produced by the script.

\subsection{Terminating a script}

This section explains two ways to terminate a running script. The first approach is as follows:

1. The manager sets the smRunControl object of the running script or the smLaunchcontrol object of the launch button used to start the running script to 'abort'. Setting smLaunchcontrol will abort all running scripts started from the launch button while smRunControl will only abort the running script associated with the smRuncontrol instance. 
The second way to terminate a script is to set the smRunLifeTime to zero which causes the runtime system to terminate the script with a 'lifeTimeExceeded' exit code:

1. The manager changes the value of smRunLifeTime to 0 . This causes the Script MIB implementation to abort the script because the remaining life time has expired.

Note, changing the smRunLifeTime value can also be used to increase the permitted lifetime of a running script. For example, a manager can choose to set smRunLifeTime to a small fixed time interval and increase the value periodically. This strategy has the nice effect that scripts terminate automatically if the manager loses contact with the Script MIB engine.

7.8. Removing a launch button

This section explains how a manager can remove a launch button from a distributed manager.

1. First, the manager sets the smLaunchAdminstatus to 'disabled'. This will ensure that no new scripts can be started from this launch button while running script will finish their execution.

2. The manager polls the smLaunchoperstatus object until the value is 'disabled'.

3. The manager sends an SNMP set-request to change the smLaunchRowstatus object to 'destroy'. This will remove the row and all associated resources from the script MIB implementation.

\section{VACM Configuration Examples}

This section shows how the view-based access control model defined in RFC 2275 [15] can be configured to control access to the script MIB.

\subsection{Sandbox for guests}

The first example demonstrates how to configure VACM to give the members of the VACM group "guest" limited access to the script MIB. The MIB views defined below give the members of the "guest" group a sandbox where they can install and start their own scripts, but not access any other scripts maintained by the script MIB implementation.

vacmAccessReadView."guest"." " usm.authNoPriv = "guestReadView" vacmAccessWriteView."guest"." .usm.authNoPriv = "guestWriteView" 
The guestReadView grants read access to the smLangTable, the smExtsnTable and to all the table entries owned by "guest":

guestReadView:

smLangTable (included)

smExtsnTable (included)

smScriptobjects.*.*.*."guest" (included)

smRunObjects.*.*.*."guest" (included)

The guestWriteView grants write access to all the table entries owned by "guest" :

guestWriteView:

smScriptobjects.*.*.*."guest" (included)

smRunobjects.*.*.*."guest" (included)

\subsection{Sharing scripts}

This example demonstrates how VACM can be used to share a repository of scripts between the members of the "senior" and the members of the "junior" VACM group:

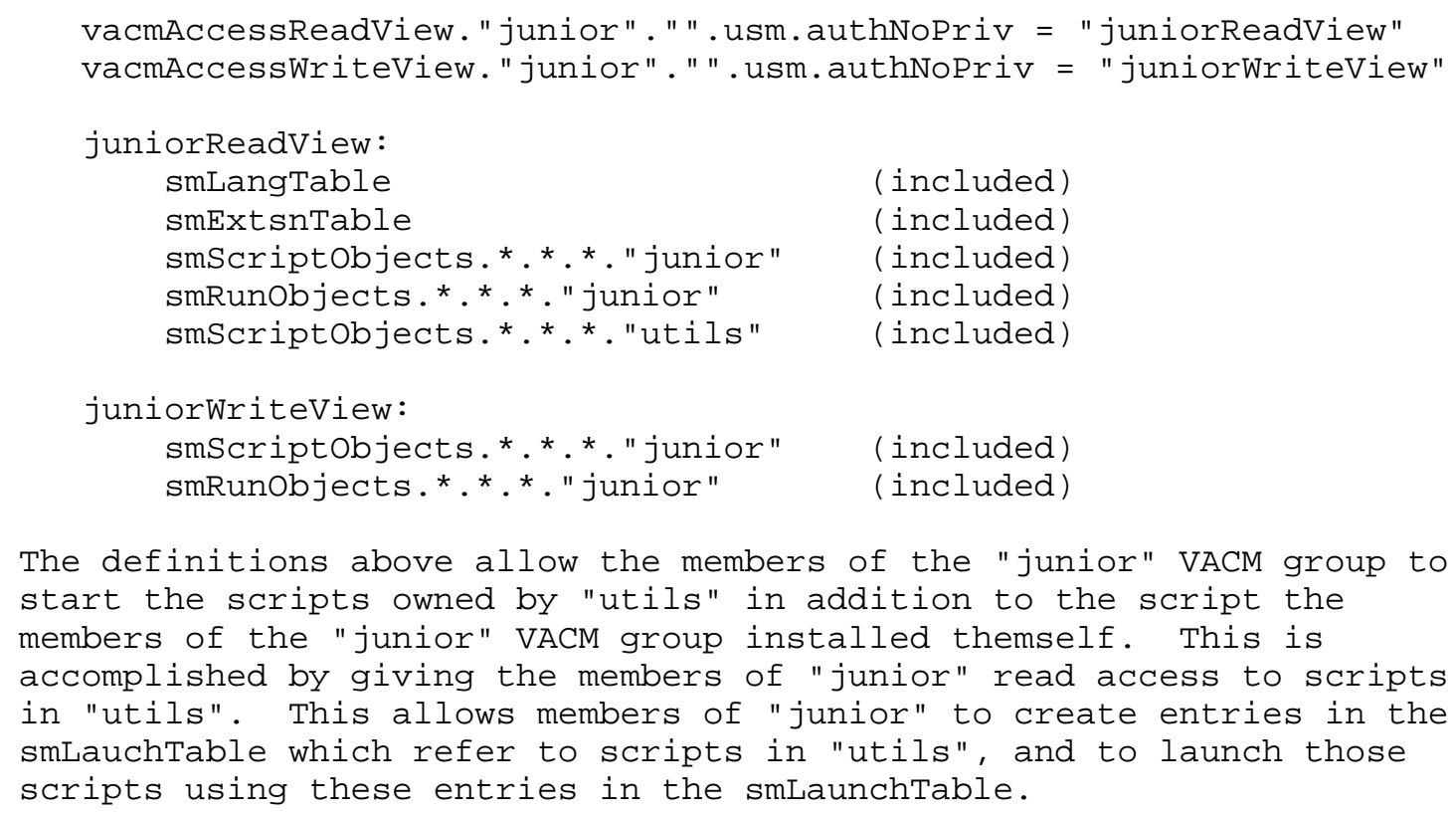

The definitions above allow the members of the "junior" VACM group to start the scripts owned by "utils" in addition to the script the members of the "junior" VACM group installed themself. This is accomplished by giving the members of "junior" read access to scripts in "utils". This allows members of "junior" to create entries in the smLauchtable which refer to scripts in "utils", and to launch those scripts using these entries in the smLaunchiable. 


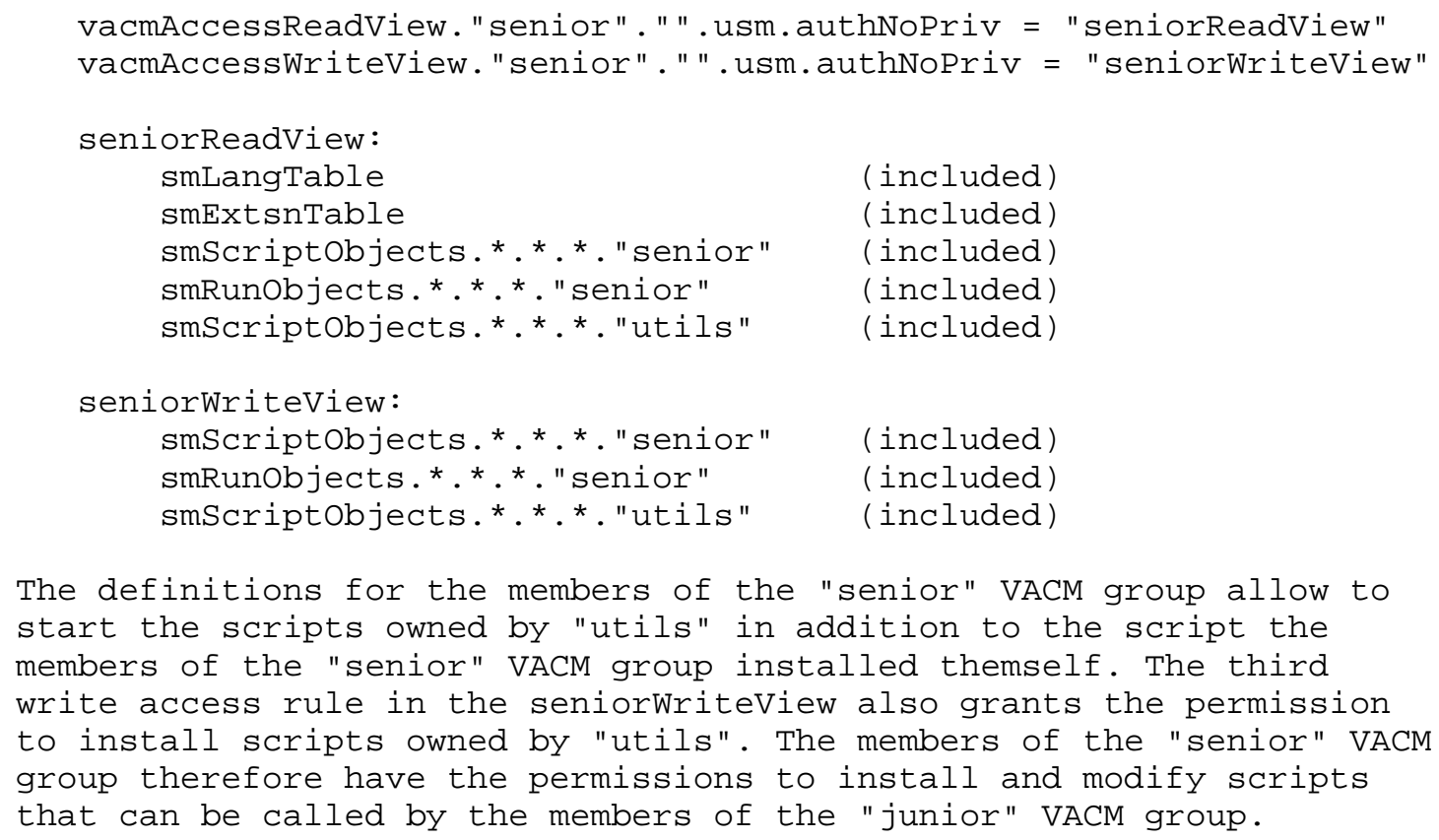

The definitions for the members of the "senior" VACM group allow to start the scripts owned by "utils" in addition to the script the members of the "senior" VACM group installed themself. The third write access rule in the seniorWriteView also grants the permission to install scripts owned by "utils". The members of the "senior" VACM group therefore have the permissions to install and modify scripts that can be called by the members of the "junior" VACM group.

8.3. Emergency scripts

This example demonstrates how VACM can be used to allow the members of the "junior" VACM group to launch scripts that are executed with the permissions associated with the "emergency" owner. This works by adding the following rules to the juniorReadView and the juniorWriteView:

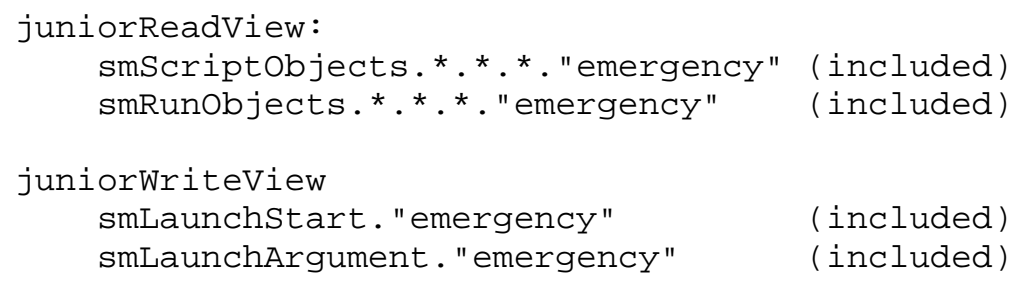

The rules added to the juniorReadView grant read access to the scripts, the launch buttons and the results owned by "emergency". The rules added to the juniorWriteView grant write permissions to the smLaunchstart and smLaunchArgument variables ownded by "emergency". Members of the "junior" VACM group can therefore start scripts that will execute under the owner "emergency". 


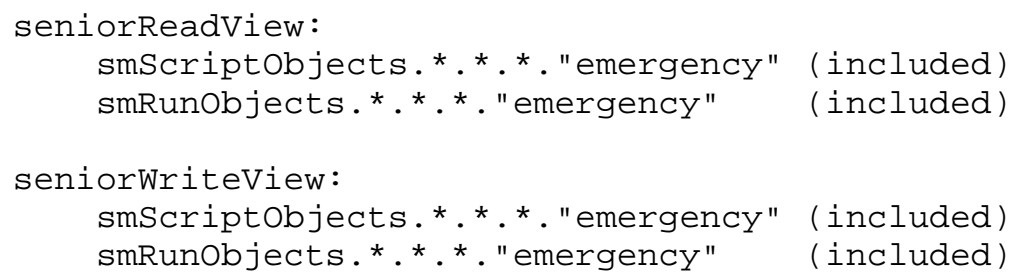

The rules added to the seniorReadView and the seniorWriteView will give the members of the "senior" VACM group the rights to install emergency scripts and to configure appropriate launch buttons.

\section{IANA Considerations}

The Internet Assigned Numbers Authority (IANA) is responsible for maintaining a MIB module which provides OID registrations for wellknown languages. The IANA language registry is intented to reduce interoperability problems by providing a single list of well-known languages. However, it is of course still possible to register languages in private OID spaces. Registering languages in private spaces is especially attractive if a language is used for experimentation or if a language is only used in environments where the distribution of MIB modules with the language registration does not cause any maintenance problems.

Any additions or changes to the list of languages registered via IANA require Designated Expert Review as defined in the IANA guidelines [20]. The Designated Expert will be selected by the IESG Area Director for the IETF Operations and Management Area.

10. Security Considerations

This MIB provides the ability to distribute applications written in an arbitrary language to remote systems in a network. The security features of the languages available in a particular implementation should be taken into consideration when deploying an implementation of this MIB.

To facilitate the provisioning of access control by a security administrator using the View-Based Access Control Model (VACM) defined in RFC 2275 [15] for tables in which multiple users may need to independently create or modify entries, the initial index is used as an "owner index". Such an initial index has a syntax of SnmpAdminstring, and can thus be trivially mapped to a securityName or groupName as defined in VACM, in accordance with a security policy. 
All entries in related tables belonging to a particular user will have the same value for this initial index. For a given user's entries in a particular table, the object identifiers for the information in these entries will have the same subidentifiers (except for the "column" subidentifier) up to the end of the encoded owner index. To configure VACM to permit access to this portion of the table, one would create vacmViewTreeFamilyTable entries with the value of vacmViewTreefamilysubtree including the owner index portion, and vacmViewTreeFamilyMask "wildcarding" the column subidentifier. More elaborate configurations are possible.

The VACM access control mechanism described above provides control over SNMP access to Script MIB objects. There are a number of other access control issues that are outside of the scope of this MIB. For example, access control on URLs, especially those that use the file scheme, must be realized by the underlying operating system. A mapping of the owner index value to a local operating system security user identity should be used by an implementation of this MIB to control access to operating system resources when resolving URLs or executing scripts.

\section{Intellectual Property}

The IETF takes no position regarding the validity or scope of any intellectual property or other rights that might be claimed to pertain to the implementation or use of the technology described in this document or the extent to which any license under such rights might or might not be available; neither does it represent that it has made any effort to identify any such rights. Information on the IETF's procedures with respect to rights in standards-track and standards-related documentation can be found in BCP-11. Copies of claims of rights made available for publication and any assurances of licenses to be made available, or the result of an attempt made to obtain a general license or permission for the use of such proprietary rights by implementors or users of this specification can be obtained from the IETF secretariat.

The IETF invites any interested party to bring to its attention any copyrights, patents or patent applications, or other proprietary rights which may cover technology that may be required to practice this standard. Please address the information to the IETF Executive Director.

\section{Acknowledgments}

This document was produced by the IETF Distributed Management (DISMAN) working group. 


\section{References}

[1] Harrington, D., Presuhn, R. and B. Wijnen, "An Architecture for Describing SNMP Management Frameworks", RFC 2271, January 1998.

[2] Rose, M. and K. McCloghrie, "Structure and Identification of Management Information for TCP/IP-based Internets", STD 16, RFC 1155, May 1990 .

[3] Rose, M. and K. McCloghrie, "Concise MIB Definitions", STD 16 , RFC 1212, March 1991.

[4] Rose, M., "A Convention for Defining Traps for use with the SNMP", RFC 1215, March 1991.

[5] McCloghrie, K., Perkins, D., Schoenwaelder, J., Case, J., Rose, M. and S. Waldbusser, "Structure of Management Information Version 2 (SMIv2)", STD 58, RFC 2578, April 1999.

[6] McCloghrie, K., Perkins, D., Schoenwaelder, J., Case, J., Rose, M. and S. Waldbusser, "Textual Conventions for SMIv2", STD 58, RFC 2579, April 1999.

[7] McCloghrie, K., Perkins, D., Schoenwaelder, J., Case, J., Rose, M. and S. Waldbusser, "Conformance Statements for SMIv2", STD 58, RFC 2580, April 1999.

[8] Case, J., Fedor, M., Schoffstall, M. and J. Davin, "Simple Network Management Protocol", STD 15, RFC 1157, May 1990.

[9] Case, J., McCloghrie, K., Rose, M. and S. Waldbusser, "Introduction to Community-based SNMPv2", RFC 1901, January 1996.

[10] Case, J., McCloghrie, K., Rose, M. and S. Waldbusser, "Transport Mappings for Version 2 of the Simple Network Management Protocol (SNMPv2) ", RFC 1906, January 1996.

[11] Case, J., Harrington D., Presuhn R. and B. Wijnen, "Message Processing and Dispatching for the Simple Network Management Protocol (SNMP)", RFC 2272, January 1998.

[12] Blumenthal, U. and B. Wijnen, "User-based Security Model (USM) for version 3 of the Simple Network Management Protocol (SNMPv3)", RFC 2274, January 1998. 
[13] Case, J., McCloghrie, K., Rose, M. and S. Waldbusser, "Protocol Operations for Version 2 of the Simple Network Management Protocol (SNMPv2)", RFC 1905, January 1996.

[14] Levi, D., Meyer, P. and B. Stewart, "SNMPv3 Applications", RFC 2273, January 1998.

[15] Wijnen, B., Presuhn, R. and K. McCloghrie, "View-based Access Control Model (VACM) for the Simple Network Management Protocol (SNMP) ", RFC 2275, January 1998.

[16] Hovey, R. and S. Bradner, "The Organizations Involved in the IETF Standards Process", BCP 11, RFC 2028, October 1996.

[17] Berners-Lee, T., Fielding, R. and L. Masinter, " Uniform Resource Identifiers (URI): Generic Syntax", RFC 2396, August 1998 .

[18] Postel, J. and J. Reynolds, "File Transfer Protocol", STD 9, RFC 959, October 1985 .

[19] Fielding, R., Gettys, J., Mogul, J., Frystyk, H. and T. Berners-Lee, "Hypertext Transfer Protocol -- HTTP/1.1", RFC 2068, January 1997 .

[20] Narten, T. and H. Alvestrand, "Guidelines for Writing an IANA Considerations section in RFCs", BCP 26, RFC 2434, October 1998.

[21] Bradner, S., "Key words for use in RFCs to Indicate Requirement Levels", BCP 14, RFC 2119, March 1997. 


\section{Editors' Addresses}

David B. Levi

Nortel Networks

4401 Great America Parkway

Santa Clara, CA 95052-8185

U.S.A.

Phone: +1 4236860432

EMail: dlevianortelnetworks.com

Juergen Schoenwaelder

TU Braunschweig

Bueltenweg $74 / 75$

38106 Braunschweig

Germany

Phone: +49531 391-3683

EMail: schoenwaibr.cs.tu-bs.de 
16. Full Copyright statement

Copyright (C) The Internet Society (1999). All Rights Reserved.

This document and translations of it may be copied and furnished to others, and derivative works that comment on or otherwise explain it or assist in its implementation may be prepared, copied, published and distributed, in whole or in part, without restriction of any kind, provided that the above copyright notice and this paragraph are included on all such copies and derivative works. However, this document itself may not be modified in any way, such as by removing the copyright notice or references to the Internet society or other Internet organizations, except as needed for the purpose of developing Internet standards in which case the procedures for copyrights defined in the Internet Standards process must be followed, or as required to translate it into languages other than English.

The limited permissions granted above are perpetual and will not be revoked by the Internet society or its successors or assigns.

This document and the information contained herein is provided on an "AS IS" basis and THE INTERNET SOCIETY AND THE INTERNET ENGINEERING TASK FORCE DISCLAIMS ALL WARRANTIES, EXPRESS OR IMPLIED, INCLUDING BUT NOT LIMITED TO ANY WARRANTY THAT THE USE OF THE INFORMATION HEREIN WILL NOT INFRINGE ANY RIGHTS OR ANY IMPLIED WARRANTIES OF MERCHANTABILITY OR FITNESS FOR A PARTICULAR PURPOSE.

Acknowledgement

Funding for the RFC Editor function is currently provided by the Internet society. 